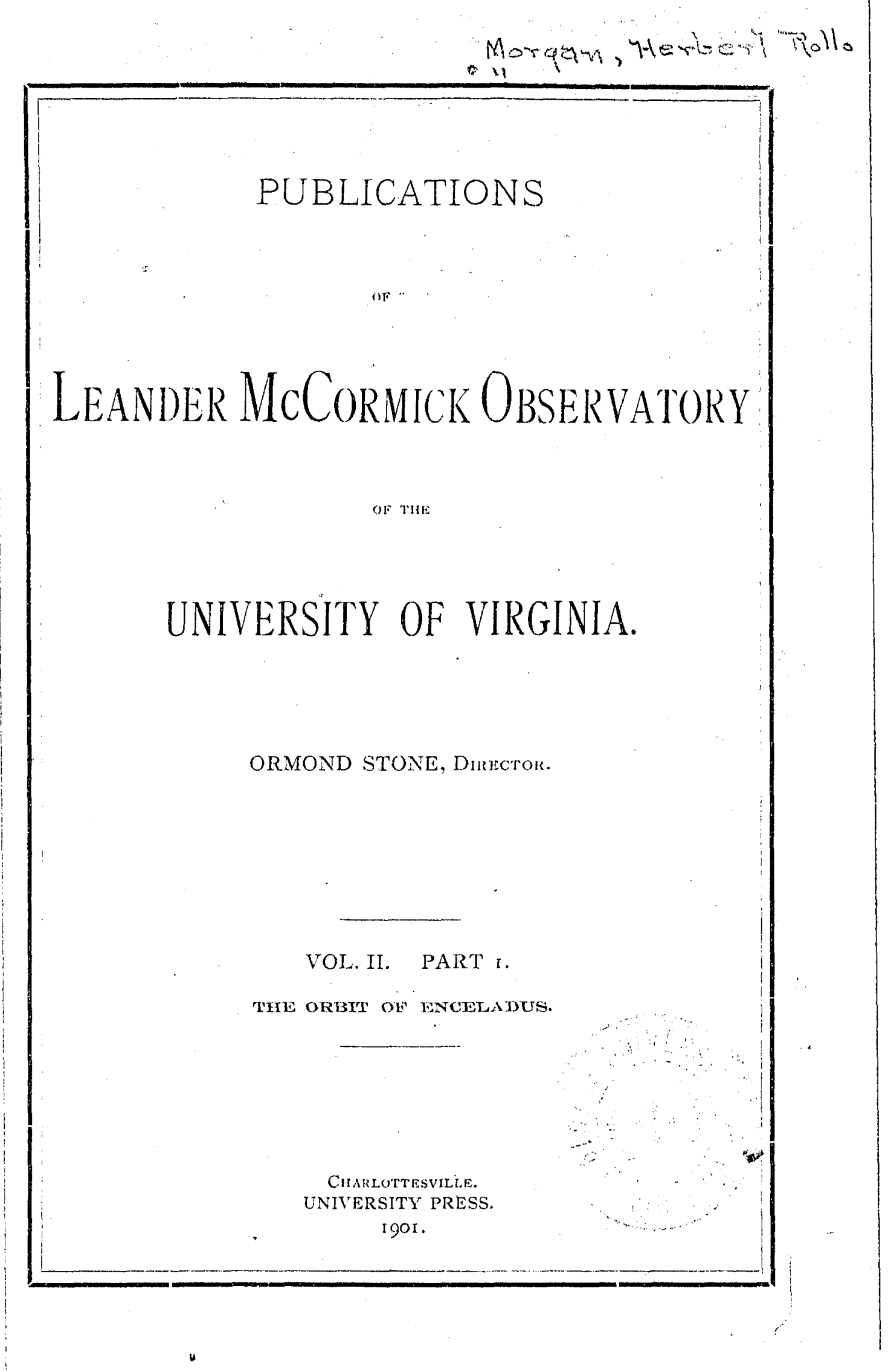


THE ORBIT OF ENCELADUS. 


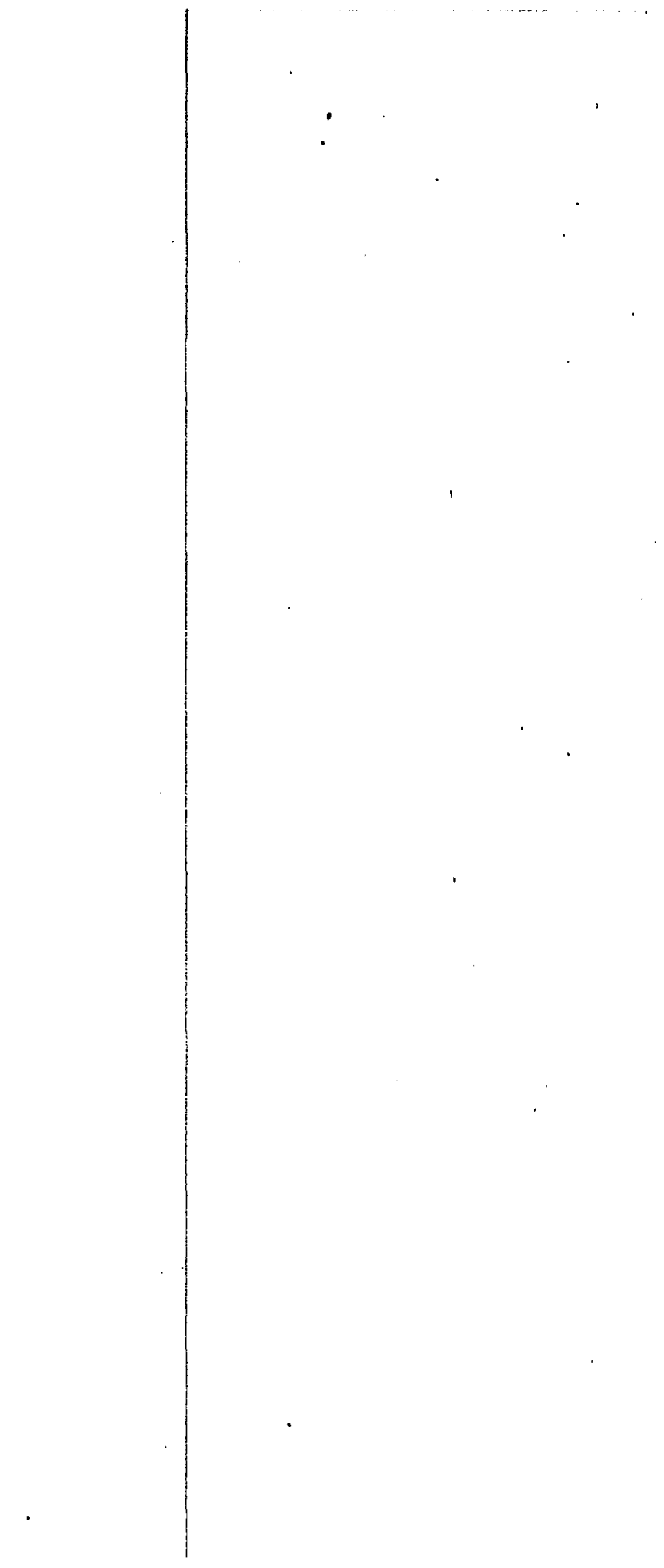




\section{THE ORBIT OF ENCELADUS.}

BY HERBERT R. MORGAN.

Having been engaged in observing the Satellites of Saturn for a number of years, $I$ became interested in reducing the observations made at this observatory, and this paper contains some of the results. Orbits of Mimas, Rhea, and Titan have been computed, but there are more observations of these satellites to be reduced.

Enceladus, the second satellite of Saturn, was discovered in 1789 by Sir William Herschel. Its revolution around Saturn is made in thirtythree hours, at a mean distance of one hundred and fifty-seven thousand miles. Althongh small and quite faint, and, moreover, close to the rings, Enceladus can be followed to conjunction on fair nights with the 26-in. refinctor withont much difficulty.

The interest in this satellite, as pointed out by H. Struve, centers in the fact that its mean motion is nearly twice the mean motion of Dione, the fourth satellite. The perturbations arising from this relation give a libration in mean lougitude, and motions of the node and perisaturnium of Enceladus.

'The formulin used in the reductions are those given by H. Struve in his work on the satellites, pp. 55-62," and the symbols have the following meaning :

$E$, mean longitude, reckoned trom the Vernal equinox on the ecliptic.

$P$, lougitude of perisaturnium, reckoned from the Vernal equinox on the ecliptic.

$I, N$, inclination and node on Earth's equator, reckoned from the Vernal equinox on the equator.

$\gamma, \theta$, inclination and node on Suturn's equator, reckoned from node of Saturn's equator on Earth's equator.

$\pi$, longitude of perisatumnium, reckoned as $\theta$.

$e$, eccentricity.

$n$, mean daily motion.

* Publicatious de Pulkovo, Séries II, Vol. XI. 
$\triangle$, mean distance from Satmrn at Saturn's mean distance from earth, $\log (p)=0.97950$.

$x_{i}, y_{i}$, ccördinates of satellite referred to Saturn's center.

The assumed elements were also derived from the same work, with the exception of the longitudes for 1898 aud 1900 . In these years in preliminary solution of the equations of condition give the longitudes both of Enceladus and of the comparison satellites. In these years the elements of the comparison satellite were taken is known, and the observations combined into one solution for Enceladus.

At Prof. Stone's sugrestion the mass of Satum, $m=\frac{1}{3500}$, was taken as a constant, and terms introdnced in the equations for the correction to the micrometer screw $M=9^{\prime \prime} .900$ of the form $\left(x_{1}-x_{2}\right) \frac{d M}{d K}$ for the equations in $x_{1}-x_{2}$, and $\left(y_{1}-y_{2}\right) \frac{d \cdot M}{d M}$ for the equations in $y_{1}-y_{2}$. These terms have a different sign for 1894 than for the later years, and the correction for this year was therefore made subtractive.

The orbits for 1894 are referred to the Earth's equator, aud those of 1898 and 1900 to Satru's equator as the fundamental planes.

The observations in 1894 were micle by Professors Stoue and Juvett, and are published in the Astronomical Journal, Vol. XV, p. 110, and Astronomische Nachuichten, Vol. 143, p. 291, respectively. Those in 1898 and 1900 were made by myself, and are published in the Astronomical Journal, Vol. XIX, p. 117, aud Astronomische Nachrichten, Vol. 154, p. 91. In 1894 and 1898 four, and some times only two, measures of angle and distance were made on a night. In 1900 eight measures of each were made. The ratio of the probable errol's of an equation should, therefore, be greater than $1 / 2: 1$. The results show this.

I wish to express my sincere thanks to Prof. Stone for his kind encouragement and valuable suggestions in all $m y$ work at the observatory, and especially in this. Also, I wish to acknowledge my indebtedness to Mr. E. R. Jones for his assistance in the least square solution of the last two orbits. Mr. Jones' work was afterwards clecked by me. 
BY HERBERT R. MORGAN.

Assuned Elements.

Enceludus. T'ethys. Dione. Thea.

Epoct : May 8.0 1894. G. M. T.

$\begin{array}{lllll}E & 331^{\circ} .900 & 66^{\circ} .595 & 275^{\circ} .059 & 220^{\circ} .748\end{array}$

$\begin{array}{lllll}I^{2} & \overline{0.0} & \overline{0.0} & \overline{0.0} & \overline{0.0}\end{array}$

$\left.I \quad 6^{\circ} 53^{\prime} 34^{\prime \prime} \quad 6^{\circ} 53^{\prime} 34^{\prime \prime} \quad 6^{\circ} 53^{\prime} 34^{\prime \prime} \quad 6^{\circ} 53^{\prime} 34^{\prime \prime}\right\}$ Apparent equator,

$\begin{array}{llllll}N & 1253436 & 1253336 & 1253436 & 1253436\} & 1894.35 .\end{array}$

$\begin{array}{lllll}n . & 262^{\circ} .7318 & 1 ! 10^{\circ} .6980 & 131^{\circ} .5349 & 79^{\circ} .6901\end{array}$

$\Delta \quad 34^{\prime \prime} .38$ 48".583 $54^{\prime} .540 \quad 76^{\prime \prime} .170$

Sitturn's equator: $\left\{N_{1}=125^{\circ} 34^{\prime} 36\right.$

1894.35. $\quad\left\{l_{1}=65334\right.$

Eroch: July 8.0 1898. G. M. T.

$\begin{array}{lllll}E^{\prime} & 249^{\circ} .557 & 149^{\circ} .180 & 312^{\circ} .014 & 190^{\circ} .258\end{array}$

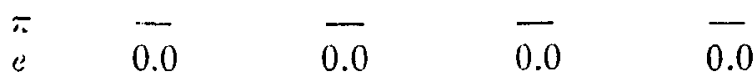

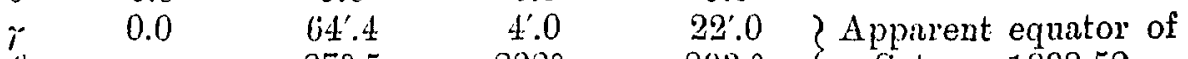

() $\left.\quad-\quad 27^{\circ} .5 \quad 222^{\circ} \quad 293 .^{\circ}\right\}$ Siaturu, 1898.52 .

"1 $262^{\circ} .7318 \quad 190^{\circ} .6980 \quad 131^{\circ} .5349679^{\circ} .6901$

C $34^{\prime \prime} .38 \quad 42^{\prime \prime} .583 \quad 54^{\prime \prime} .540 \quad 76^{\prime \prime} .170$

Sicturn's equator: $\left\{N_{1}=125^{\circ} 54^{\prime} .4\right.$

189)8.52. $\quad\left\{I_{1}=657.0\right.$

Eroch : July 8.0 1900, G. M. T.

$\begin{array}{lllll}E & 164^{\circ} .910 \quad 35^{\circ}\left(020 \quad 213^{\circ} .264\right. & 44^{\circ} .058\end{array}$

$\begin{array}{lllll}\bar{e} & \overline{0.0} & \overline{0.0} & \overline{0.0} & \overline{0.0}\end{array}$

$\gamma \quad 0.0 \quad 64^{\prime} .4 \quad 4^{\prime} .0 \quad 22^{\prime} .0 \quad$ ? Apparent equator of

1) $\quad-242^{\circ} .5 \quad 160^{\circ}$. $293^{\circ}$. 3 Saturn, 1900.52 .

\# $\left.262^{\circ} .7318 \quad 190^{\circ} .6980 \quad 131^{\circ} .5349\right) \quad 79^{\circ} .6901$

$\triangle \quad 34^{\prime \prime} .38 \quad 42^{\prime \prime} .583 \quad 54^{\prime \prime} .540 \quad 76^{\prime \prime} .170$

Sitturu's equator: $\left\{N_{1}=125^{\circ} 53^{\circ} .9\right.$

1900.5\%. $\quad\left\{I_{1}=656.9\right.$ 


\begin{tabular}{|c|c|c|c|c|c|c|}
\hline $\begin{array}{l}u \\
1 \\
0\end{array}$ & $\begin{array}{l}208000 \\
0 \% 000 \\
00000 \\
11+1+\end{array}$ & 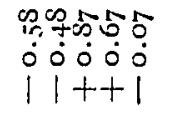 & 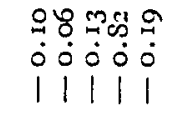 & 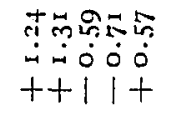 & $\begin{array}{l}3500 \% \\
00000 \\
-10000 \\
+1++1\end{array}$ & 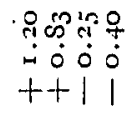 \\
\hline$\dot{0}$ & 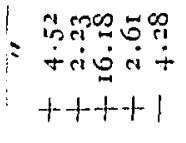 & 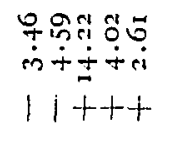 & 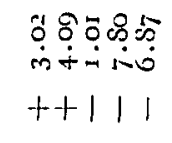 & 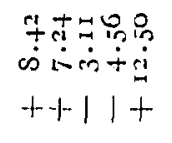 & 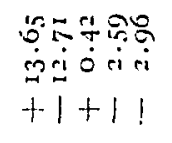 & 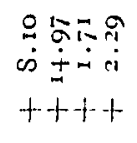 \\
\hline$\dot{\vec{c}}=$ & 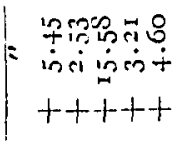 & 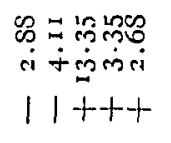 & 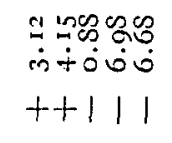 & 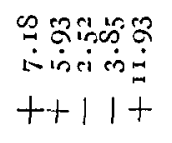 & $\begin{array}{l}80900 \overline{0} \\
89090 \\
+1111\end{array}$ & 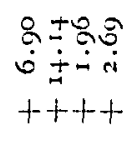 \\
\hline$\ddot{\sim}$ & 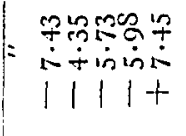 & 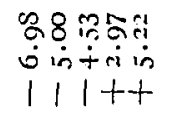 & 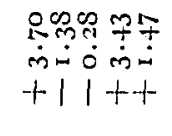 & 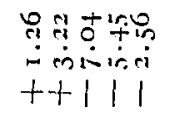 & 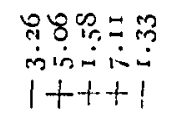 & 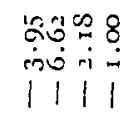 \\
\hline$\vec{\theta}$ & 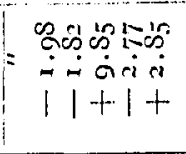 & 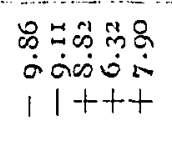 & 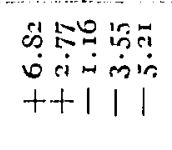 & 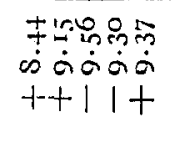 & 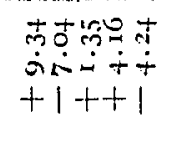 & 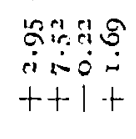 \\
\hline $\begin{array}{l}\text { U } \\
1 \\
0\end{array}$ & 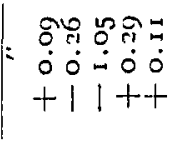 & 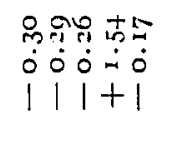 & $\begin{array}{l}150=0 \\
00000 \\
1++1+\end{array}$ & 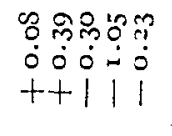 & 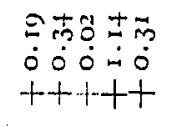 & 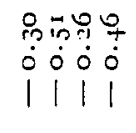 \\
\hline$\dot{\Delta} \ddot{\partial}$ & 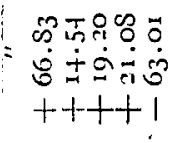 & 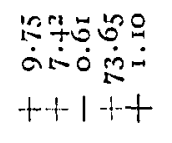 & 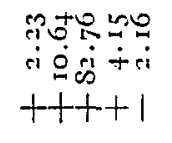 & 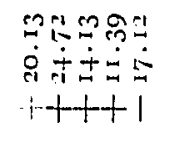 & 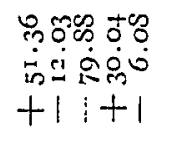 & 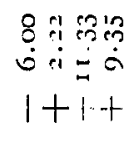 \\
\hline 官: & 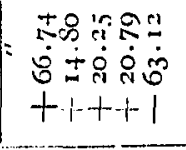 & 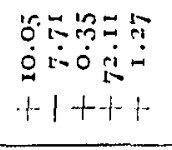 & 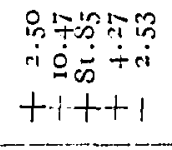 & 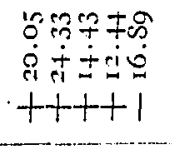 & $\begin{array}{l}15880 \\
=0800 \\
+11+1\end{array}$ & 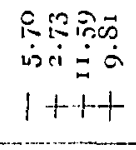 \\
\hline$\therefore$ & 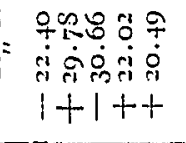 & 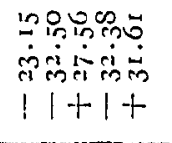 & 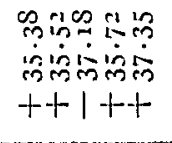 & 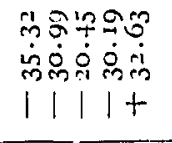 & 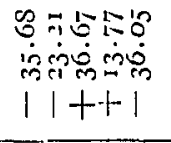 & 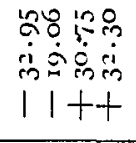 \\
\hline$\because$ & 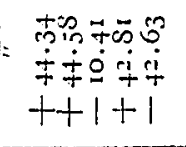 & 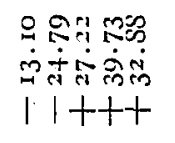 & 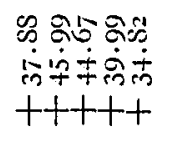 & 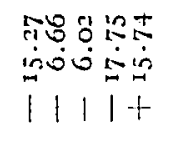 & 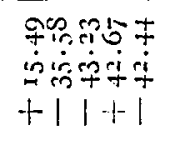 & 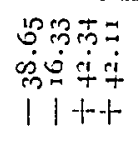 \\
\hline 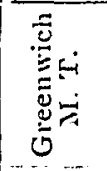 & 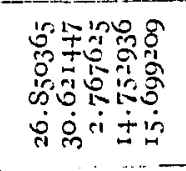 & 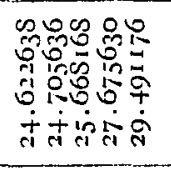 & 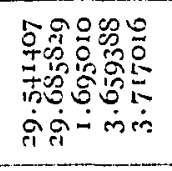 & 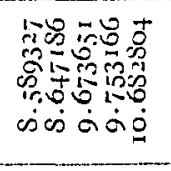 & 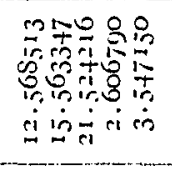 & 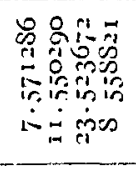 \\
\hline$\underset{\sim}{\stackrel{\hbar}{\hat{D}}}$ & 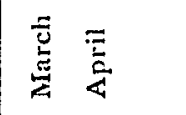 & & $\overrightarrow{\mathrm{E}}$ & · & $\stackrel{\Xi}{\Xi}$ & $\dot{\Xi}$ \\
\hline
\end{tabular}


BY HERBER'I R. MORGAN.

\begin{tabular}{|c|c|c|c|c|c|c|}
\hline 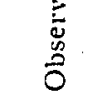 & 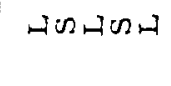 & のカカカカ & ルのカカコ & ルカカのカ & Aismang & 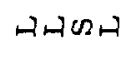 \\
\hline is & 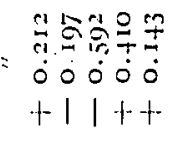 & $\begin{array}{l}\infty \infty 070 \\
08090 \\
00000 \\
+1+1+1\end{array}$ & 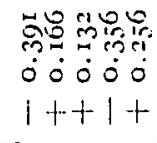 & 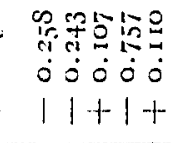 & 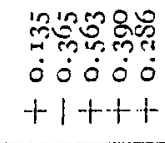 & 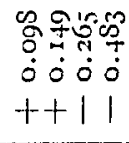 \\
\hline 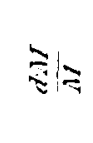 & 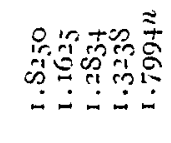 & 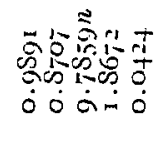 & 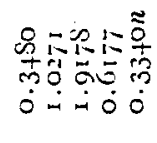 & 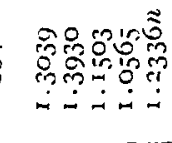 & 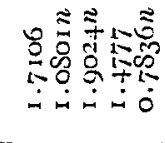 & 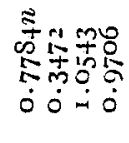 \\
\hline$\Xi$ & 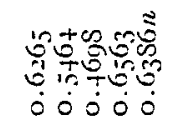 & 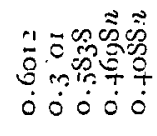 & 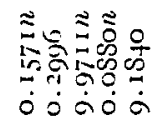 & 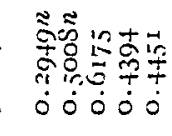 & 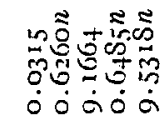 & 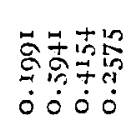 \\
\hline 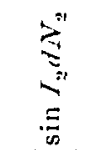 & 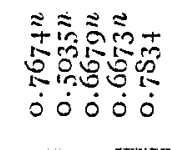 & 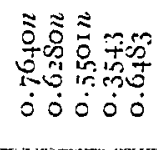 & 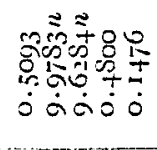 & 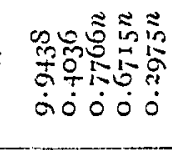 & 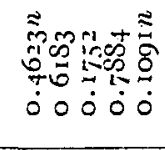 & 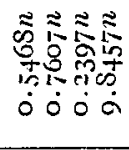 \\
\hline $\begin{array}{l}2^{n} \\
0 \\
0 \\
0 \\
0\end{array}$ & 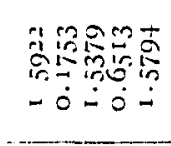 & 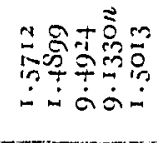 & 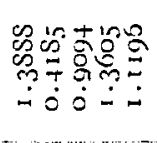 & 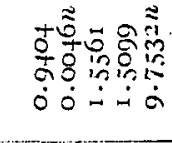 & 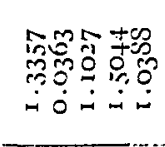 & 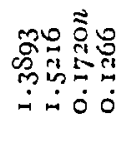 \\
\hline $\begin{array}{l}R^{\prime \prime} \\
: \Xi \\
\vdots \\
\vdots\end{array}$ & 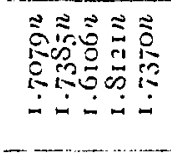 & 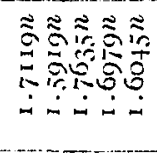 & 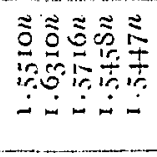 & 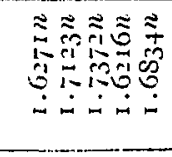 & 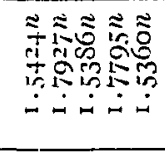 & 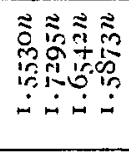 \\
\hline$\stackrel{\Delta}{*}$ & 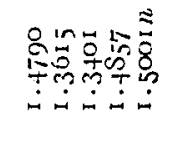 & 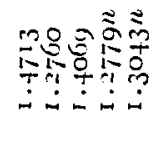 & 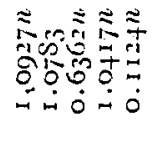 & 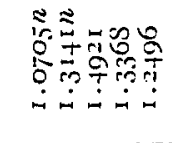 & 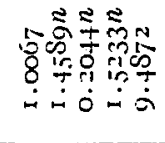 & 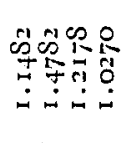 \\
\hline$\Xi$ & 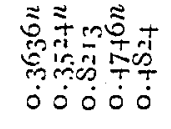 & 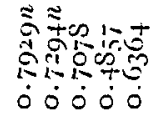 & 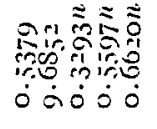 & 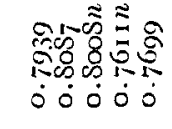 & 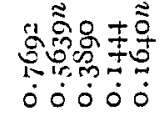 & 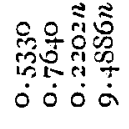 \\
\hline $\begin{array}{l}\stackrel{5}{5} \\
\stackrel{5}{5} \\
\stackrel{5}{5}\end{array}$ & 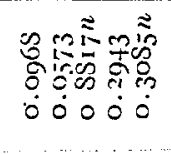 & 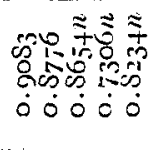 & 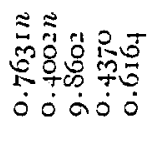 & 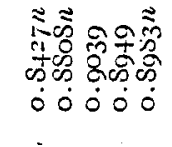 & 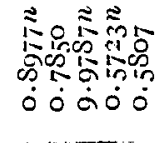 & 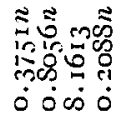 \\
\hline है & 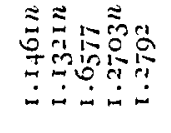 & 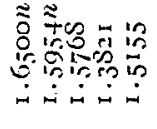 & 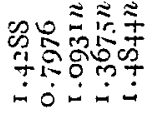 & 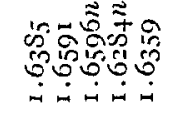 & 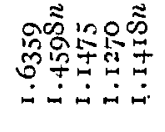 & 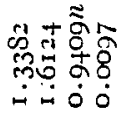 \\
\hline 壳 & 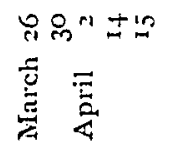 & 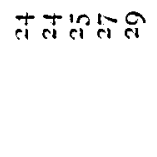 & gistmon & $\cos 000$ & 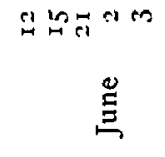 & $\begin{array}{r}n=\text { mos } \\
\quad \dot{\Xi}\end{array}$ \\
\hline
\end{tabular}


'THE ORIJI' OF ENCRLADUS.

\begin{tabular}{|c|c|c|c|c|c|c|}
\hline 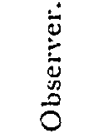 & HUA & ルカヘカへ & カルのルル & ハカルカルカ & カルロルの & カஅルேー \\
\hline is & 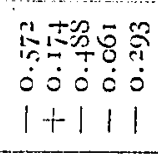 & 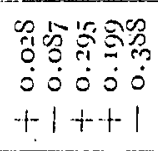 & 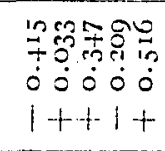 & 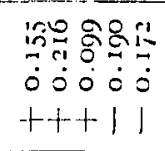 & 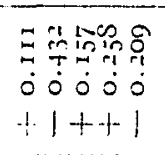 & 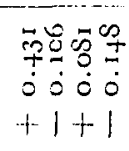 \\
\hline$\Xi$ & 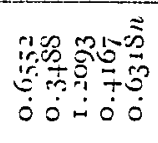 & 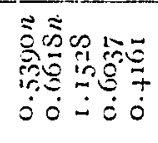 & 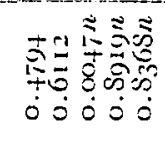 & 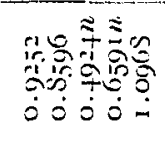 & 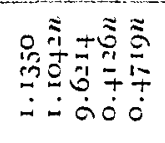 & 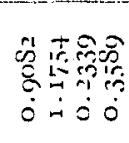 \\
\hline$\Xi$ & $\begin{array}{lll}0 \\
0 \\
0\end{array}$ & 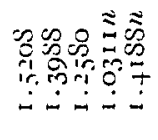 & 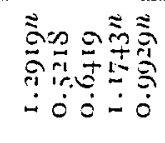 & 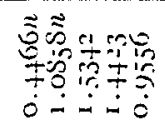 & 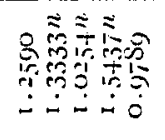 & 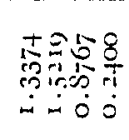 \\
\hline 言 & 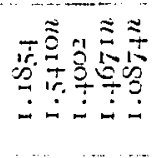 & 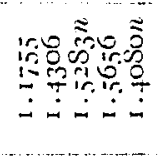 & 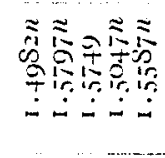 & 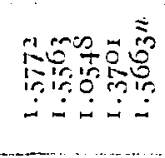 & 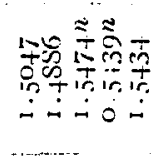 & 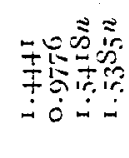 \\
\hline 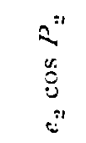 & 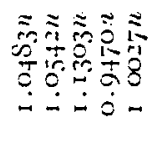 & 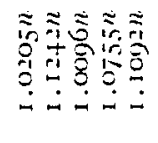 & 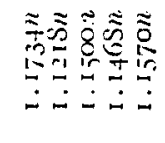 & 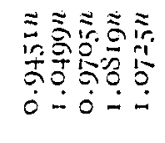 & 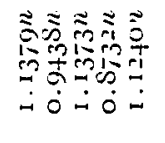 & 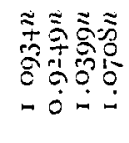 \\
\hline $\begin{array}{l}E^{\prime \prime} \\
\vdots \\
\vdots \\
\vdots \\
\vdots\end{array}$ & 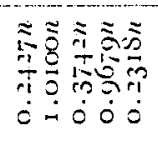 & 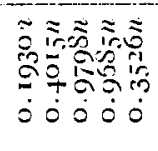 & 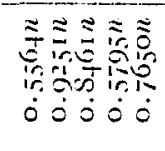 & 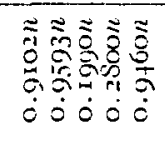 & 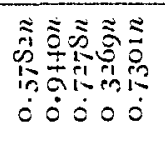 & 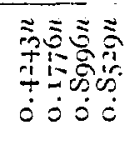 \\
\hline$\triangleq$ & 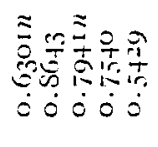 & 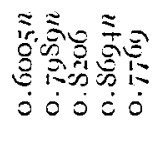 & 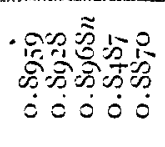 & 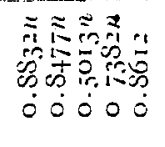 & 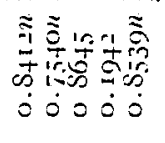 & 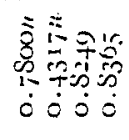 \\
\hline$\Xi$ & 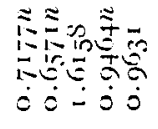 & نُ & 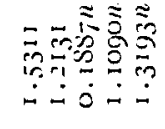 & 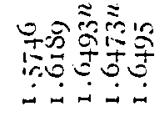 & 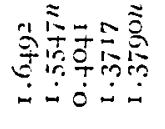 & 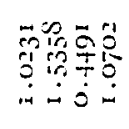 \\
\hline 三 & & 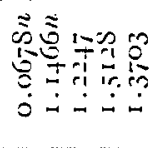 & 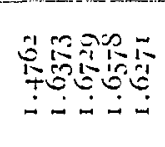 & 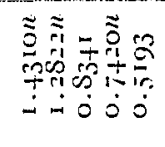 & 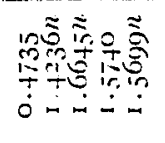 & 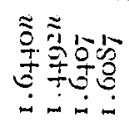 \\
\hline$\stackrel{1}{\Xi}$ & 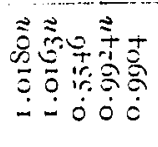 & 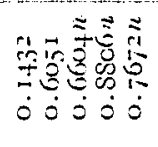 & 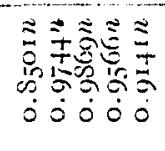 & 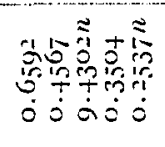 & 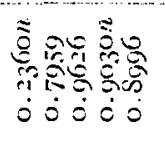 & 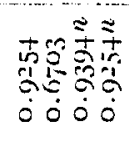 \\
\hline 离 & 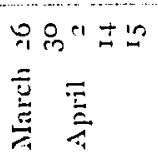 & tateisa & 至 & $\cos a \sigma^{\circ}$ & 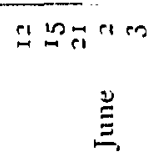 & $\begin{array}{r}\cdots=\text { as } \\
\quad \cong\end{array}$ \\
\hline
\end{tabular}


JY HEMBEHT R. MORGAN.
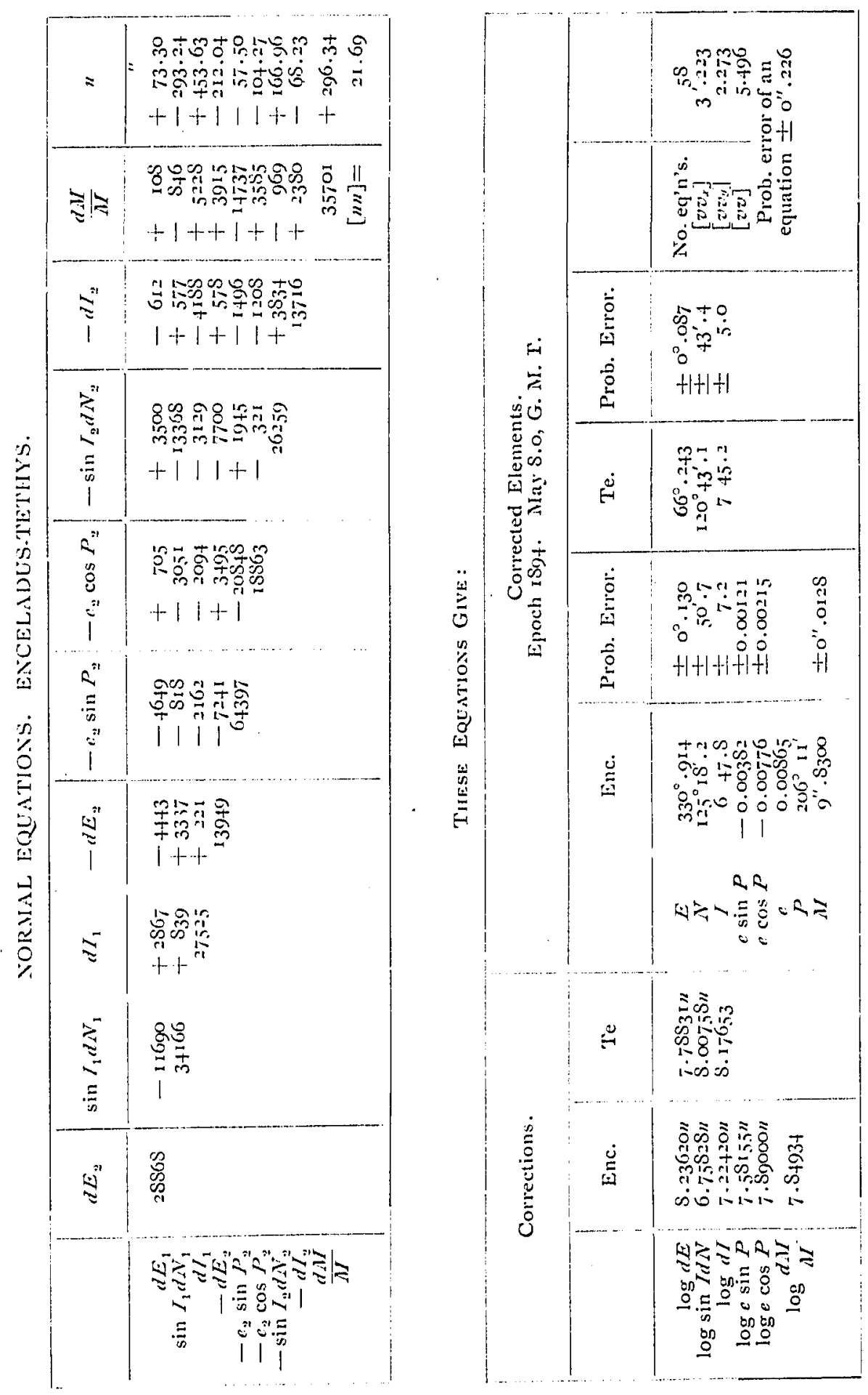


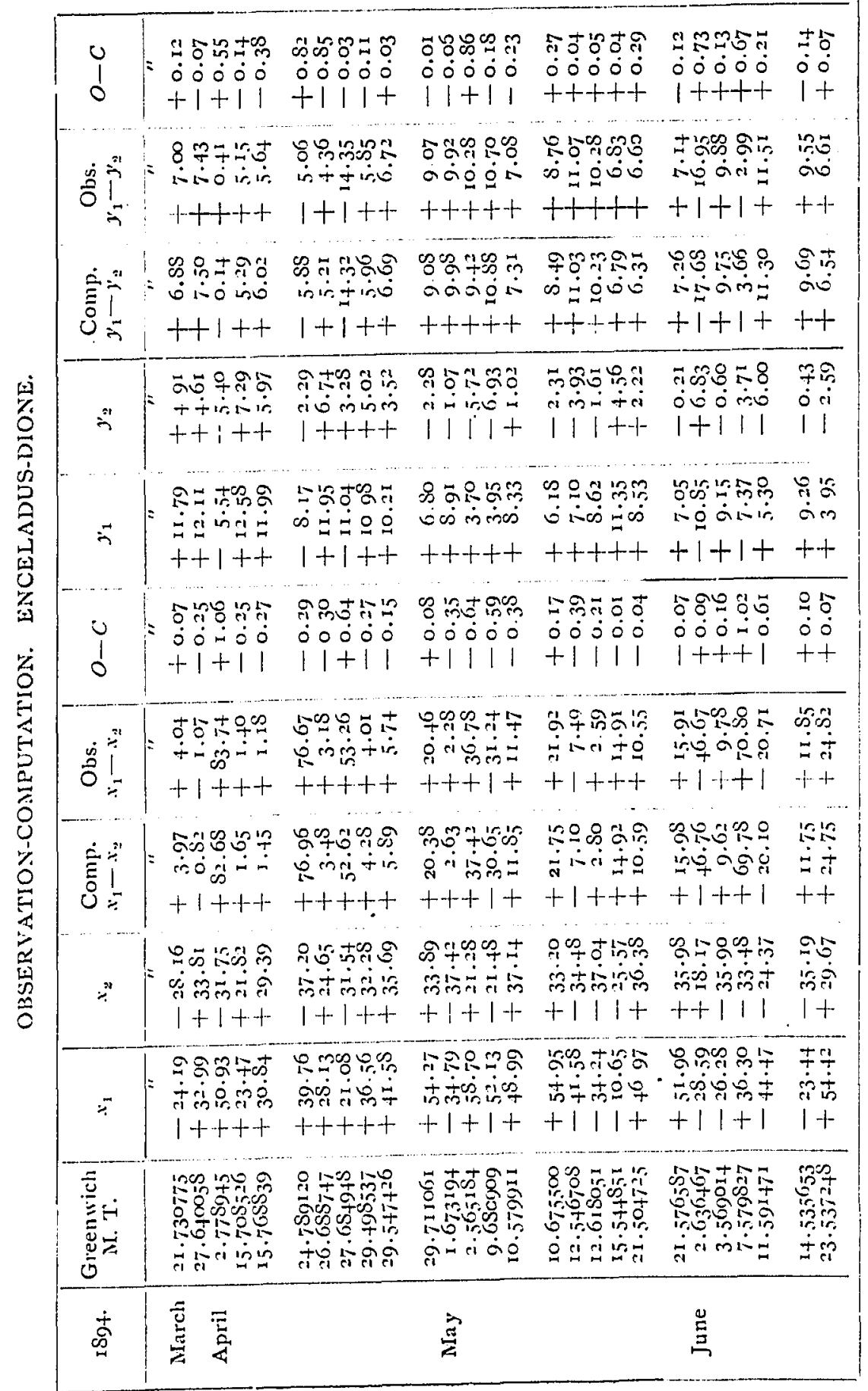




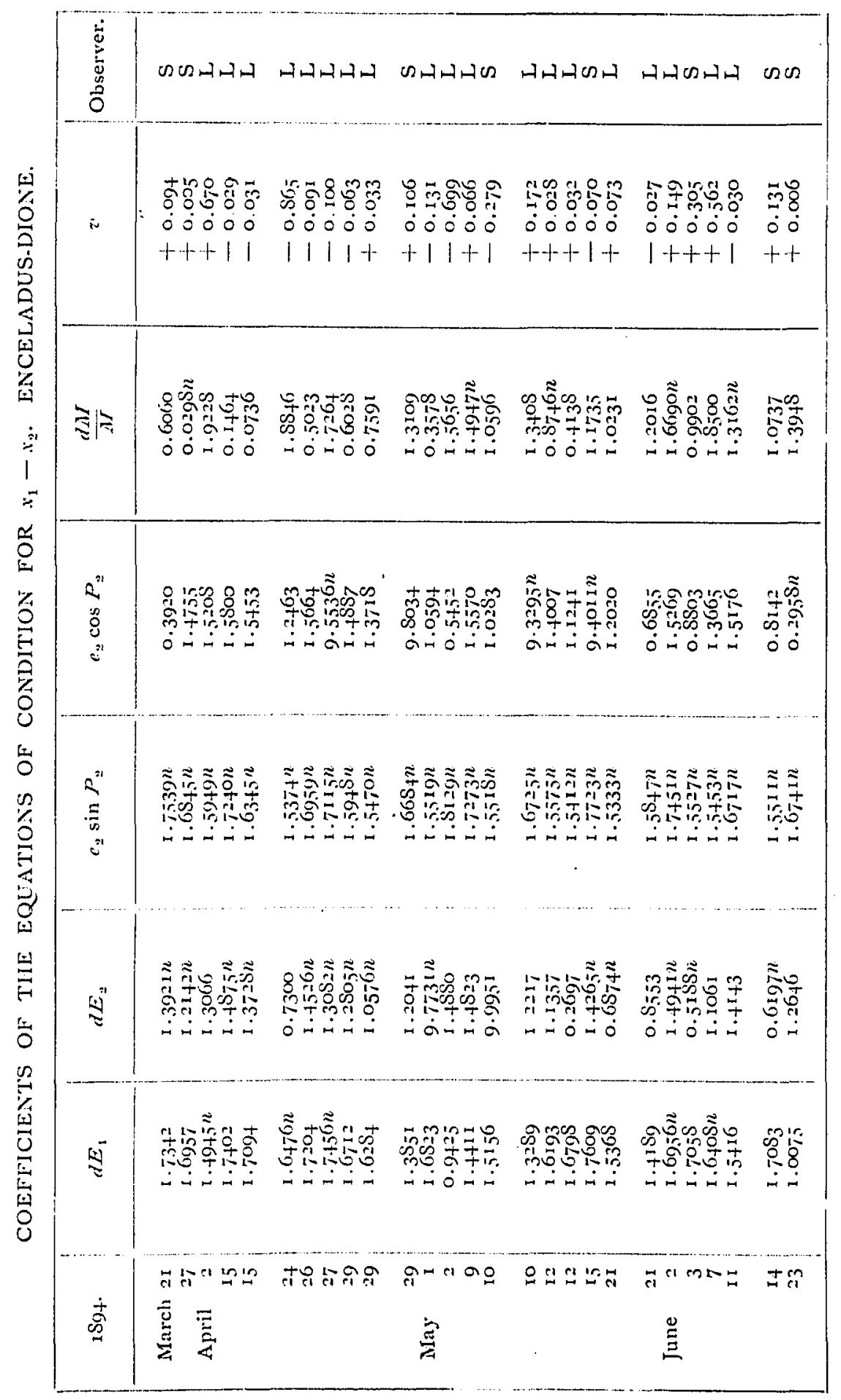


THE ORBII OF ENCELADUS.

\begin{tabular}{|c|c|c|c|c|c|c|}
\hline 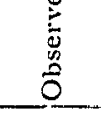 & 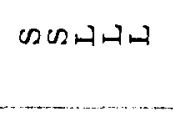 & 배 & 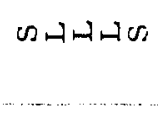 & カカカのの & $\min$ & $\cos$ \\
\hline a & $\begin{array}{l}901000 \\
08000 \\
00000 \\
++1+1\end{array}$ & 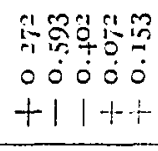 & 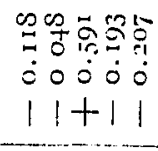 & $\begin{array}{l}070 \% \\
0000 \\
00000 \\
+1++1\end{array}$ & 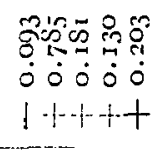 & 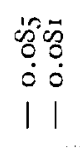 \\
\hline$\lesssim$ & 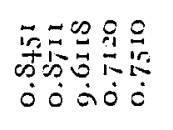 & 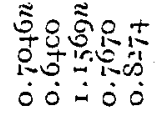 & 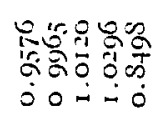 & 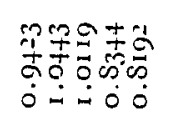 & 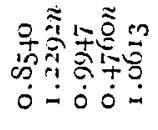 & 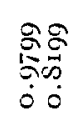 \\
\hline 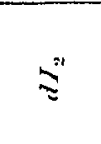 & 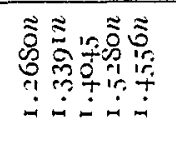 & 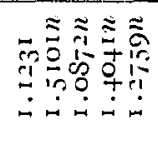 & 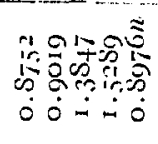 & 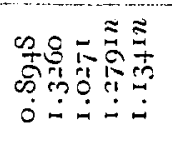 & 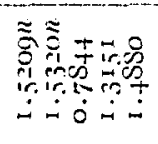 & 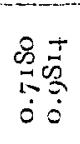 \\
\hline 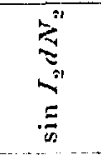 & 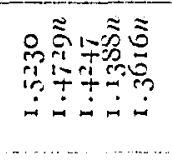 & 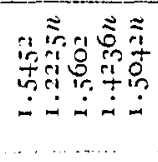 & 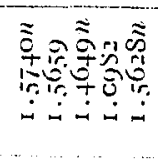 & 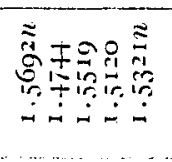 & 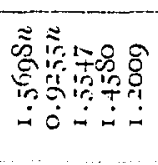 & 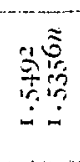 \\
\hline $\begin{array}{l}0^{n} \\
0 \\
0 \\
0 \\
0 "\end{array}$ & 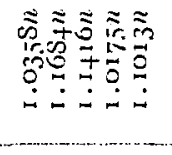 & $\begin{array}{l}0 \\
0 \\
0 \\
0\end{array}$ & 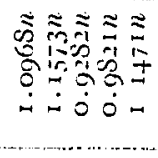 & 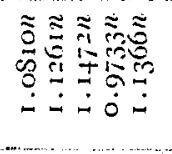 & 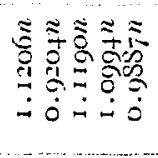 & 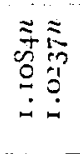 \\
\hline $\begin{array}{l}\sum^{2} \\
\vdots \\
0 \\
0 \\
0\end{array}$ & 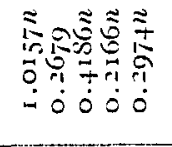 & 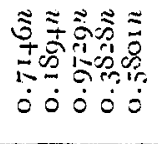 & 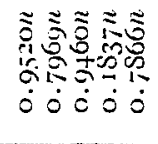 & 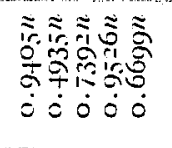 & 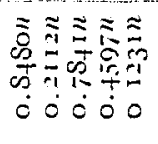 & 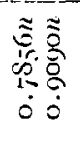 \\
\hline$\stackrel{\otimes}{\triangleq}$ & 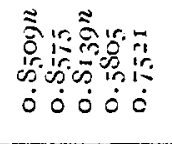 & 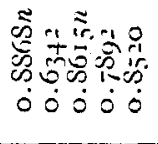 & 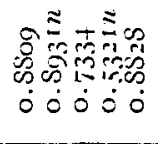 & 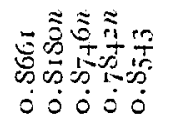 & 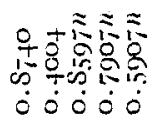 & 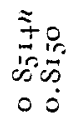 \\
\hline$\Xi$ & 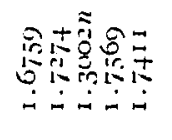 & 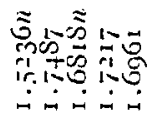 & 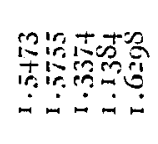 & 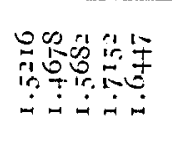 & 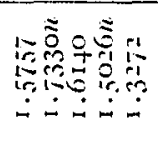 & 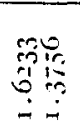 \\
\hline$\sum_{\bar{E}}$ & 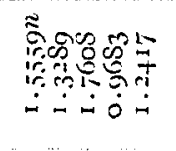 & 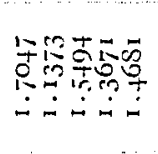 & 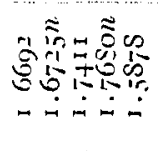 & 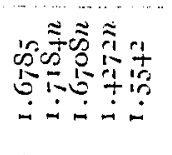 & 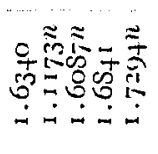 & 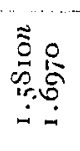 \\
\hline (2) & 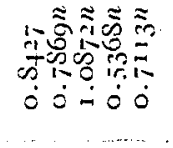 & 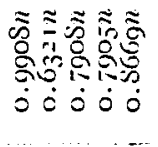 & 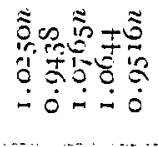 & 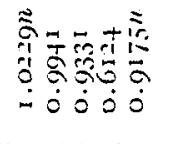 & 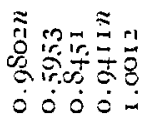 & 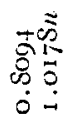 \\
\hline 畜 & 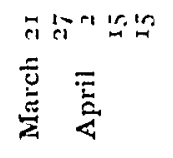 & 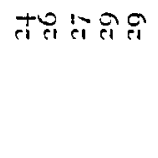 & $\begin{array}{l}\text { त }=00 \\
\text { 晋 }\end{array}$ & 욤ㅍㅁ & 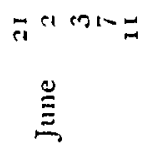 & $\pm ?$ \\
\hline
\end{tabular}


BY HERBETW T. MONGAN.
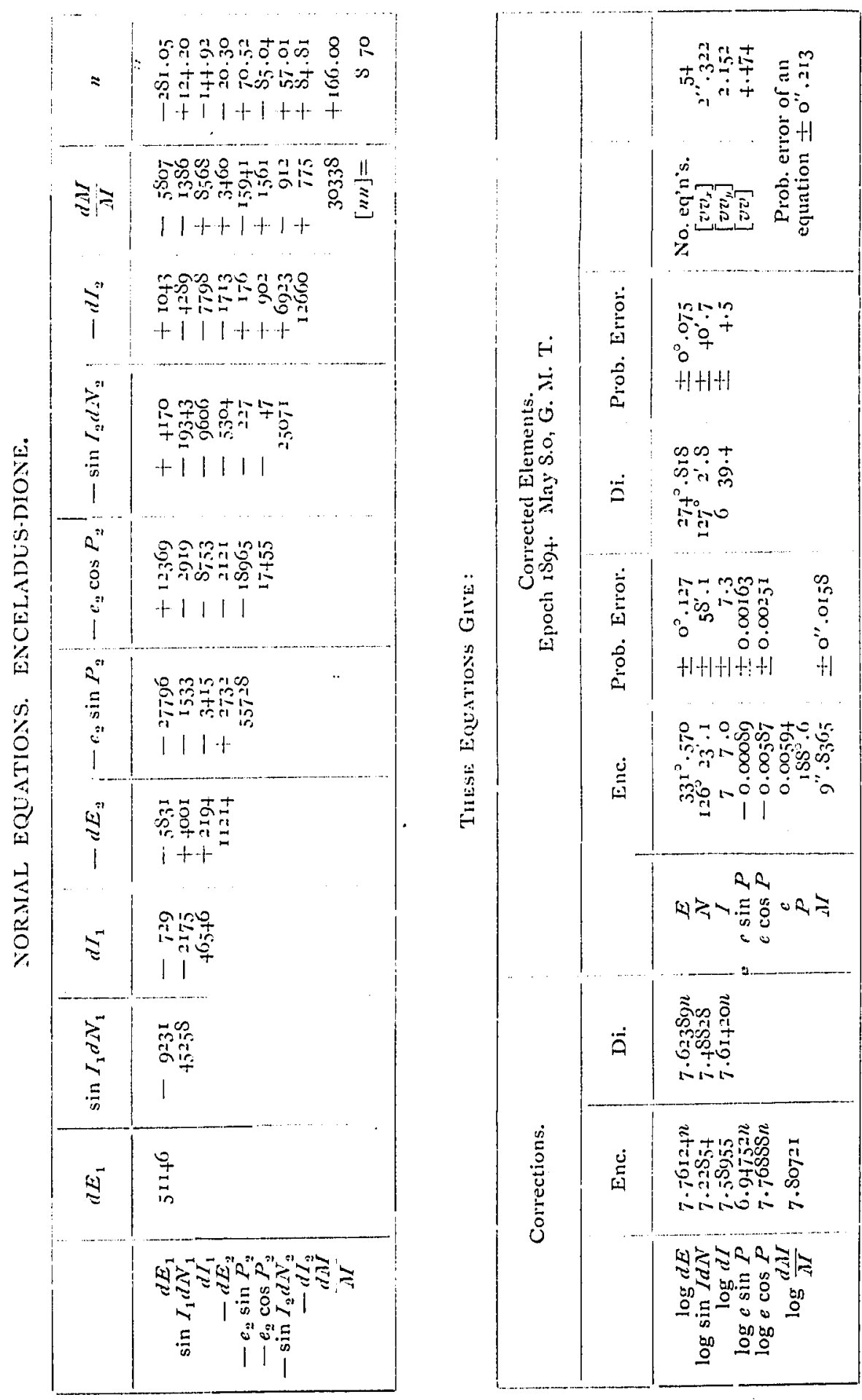
THE ORBIT OF ENOELADUS.

\begin{tabular}{|c|c|c|c|c|}
\hline $\begin{array}{l}\text { U } \\
\text { o }\end{array}$ & 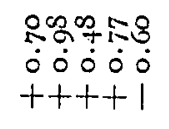 & 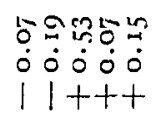 & 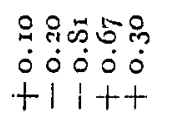 & $\begin{array}{l}700 \\
70+0 \\
000 \\
00 \\
+++1\end{array}$ \\
\hline$\tilde{0}=$ & 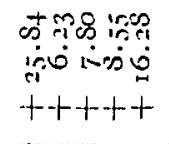 & 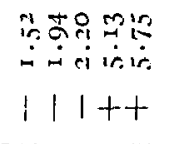 & 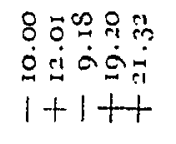 & 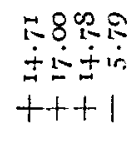 \\
\hline $\begin{array}{l}\dot{\vec{\Xi}}=\vec{i} \\
\dot{0}=\end{array}$ & 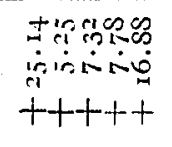 & 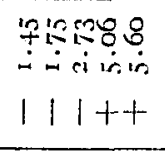 & 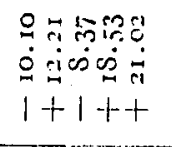 & 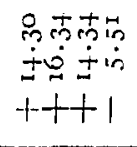 \\
\hline$\stackrel{\sim}{\sim}$ & $\begin{array}{l}\text { Serige } \\
0+\sin \\
11++1\end{array}$ & 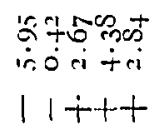 & 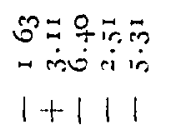 & 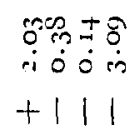 \\
\hline$=$ & 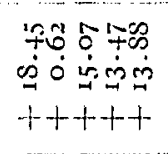 & 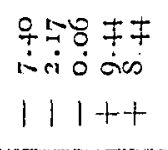 & 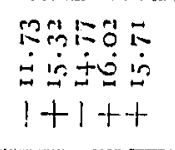 & 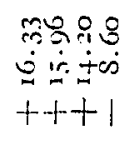 \\
\hline u & 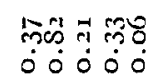 & 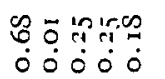 & 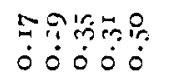 & 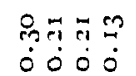 \\
\hline 0 & $1+++1$ & $+1 i++$ & $11+11$ & $11+1$ \\
\hline Oُ & 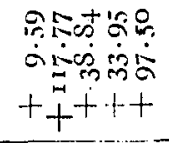 & 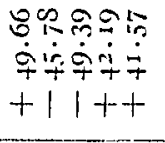 & 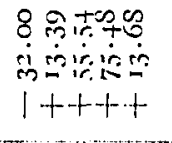 & 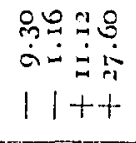 \\
\hline 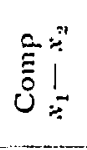 & 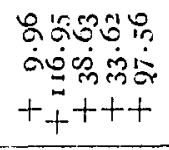 & 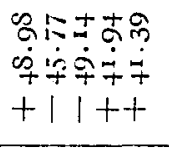 & 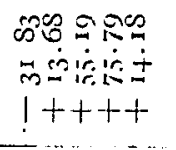 & 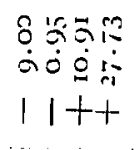 \\
\hline$\because$ & 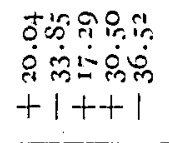 & 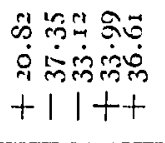 & 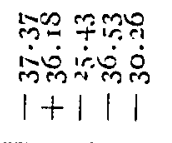 & 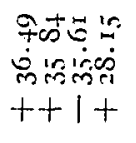 \\
\hline$\because$ & 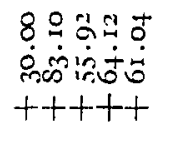 & 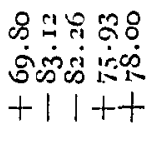 & 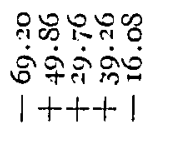 & 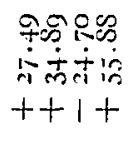 \\
\hline 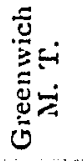 & 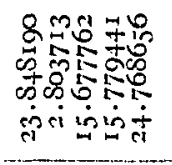 & 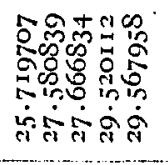 & 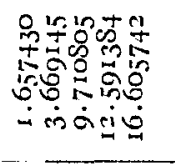 & 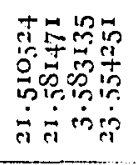 \\
\hline 壳 & \multicolumn{2}{|l|}{ 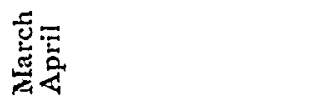 } & 密 & $\stackrel{\mathscr{U}}{\Xi}$ \\
\hline
\end{tabular}


I3Y HERBERT R. MORGAN.

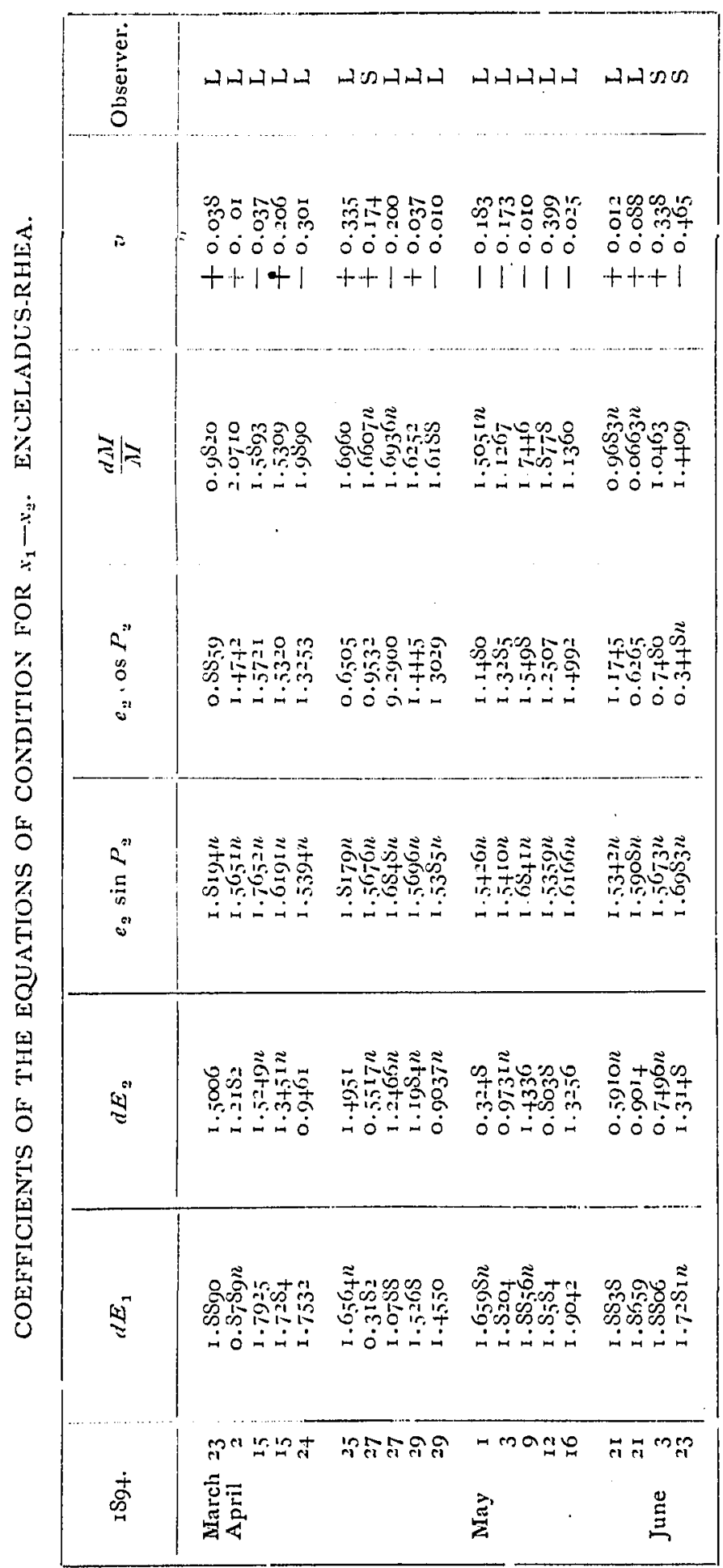


THE ORJIT OF ENCELADUS.

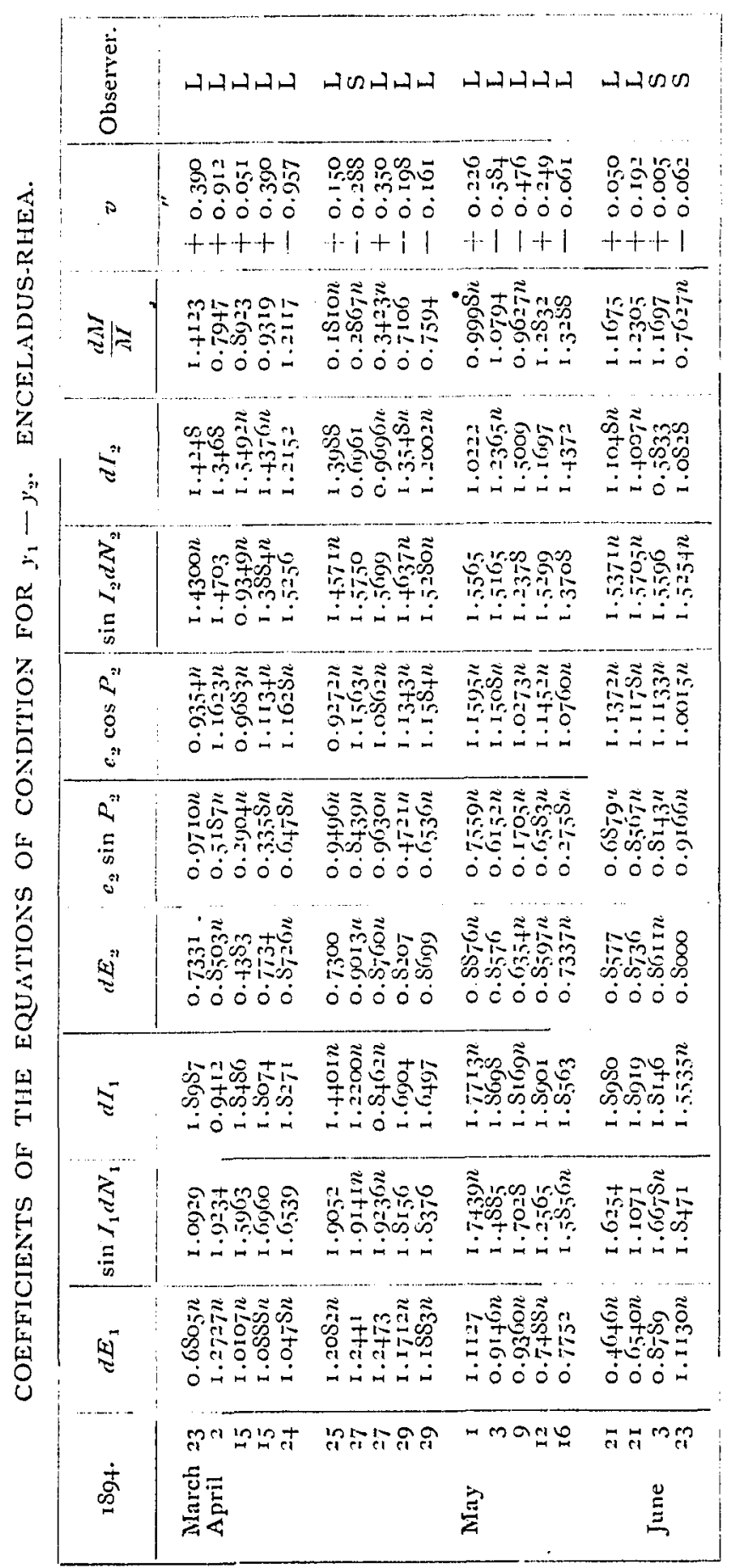


IYY HERBERT R. MLORGAN.
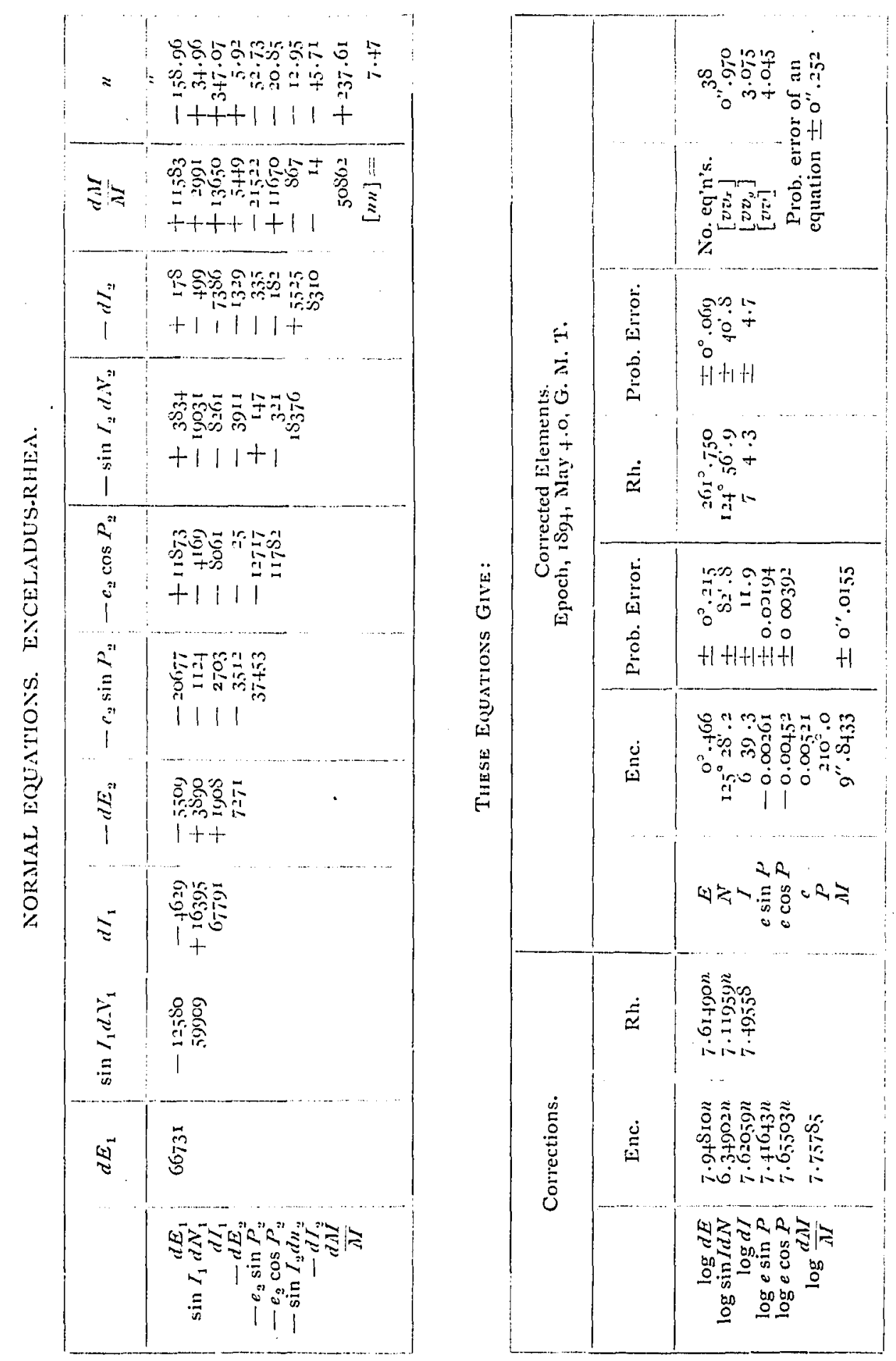


\begin{tabular}{|c|c|c|c|c|c|c|}
\hline $\begin{array}{l}0 \\
1 \\
0\end{array}$ & 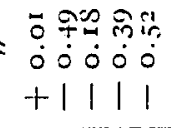 & 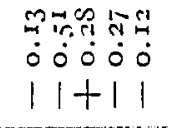 & 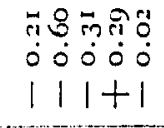 & 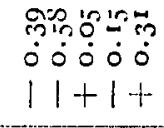 & $\begin{array}{l}91000 \\
00000 \\
+1+1+\end{array}$ & 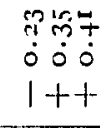 \\
\hline$\dot{\infty} \overrightarrow{0} \vec{i}$ & 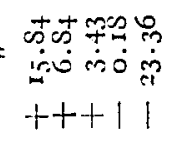 & 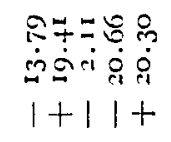 & 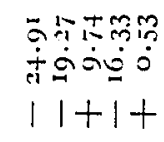 & 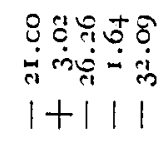 & 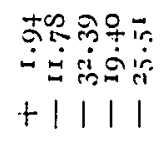 & 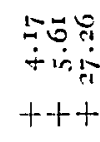 \\
\hline$\dot{\bar{\theta}}=$ & 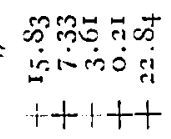 & 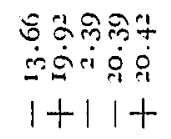 & 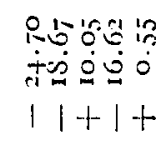 & 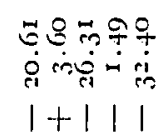 & 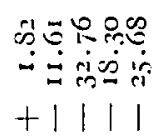 & 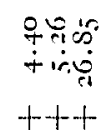 \\
\hline 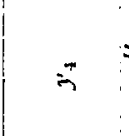 & 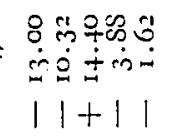 & 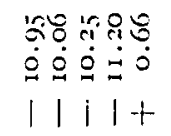 & 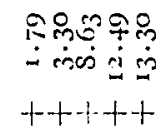 & 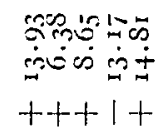 & 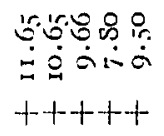 & 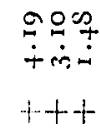 \\
\hline$\tilde{j}$ & 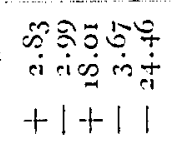 & 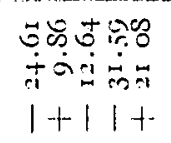 & 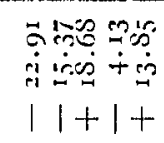 & 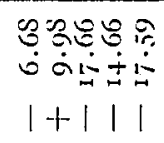 & 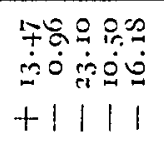 & 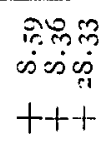 \\
\hline $\begin{array}{l}0 \\
1 \\
0\end{array}$ & 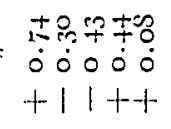 & 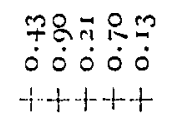 & $\begin{array}{l}20000 \\
000000 \\
111111\end{array}$ & 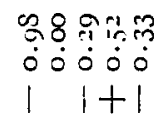 & 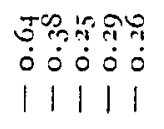 & 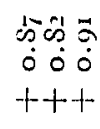 \\
\hline$\dot{\vec{u}}$ & 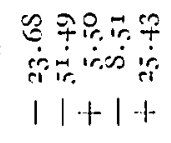 & 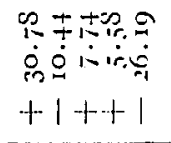 & $\begin{array}{l}21=80 \\
\pm 8+950 \\
+++1+\end{array}$ & 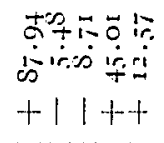 & 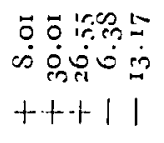 & 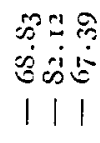 \\
\hline : & 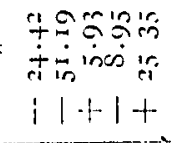 & 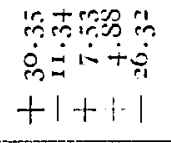 & 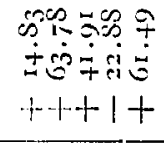 & 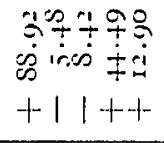 & 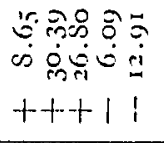 & 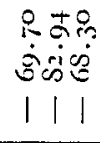 \\
\hline$\because$ & 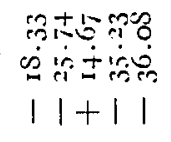 & 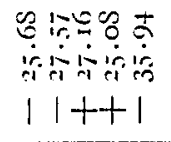 & 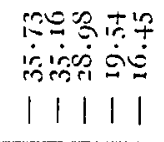 & 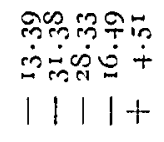 & 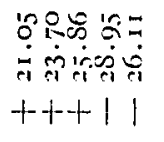 & 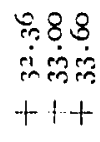 \\
\hline$\because$ & 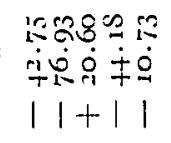 & 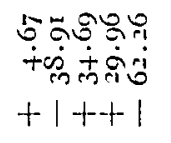 & 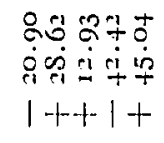 & $\begin{array}{l}\text { mos } \\
1080 \\
10000 \\
+11++\end{array}$ & 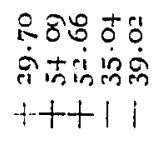 & 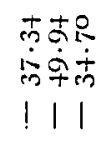 \\
\hline $\mid$ & 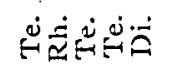 & 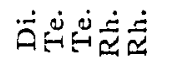 & $\dot{\vec{\Delta}} \dot{\vec{\theta}} \dot{\vec{\theta}}$ & 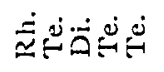 & 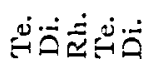 & 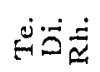 \\
\hline 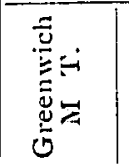 & 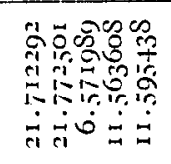 & 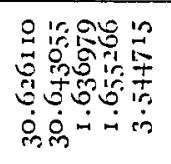 & 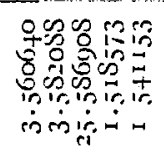 & 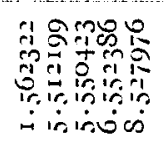 & 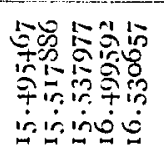 & 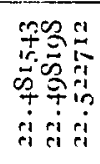 \\
\hline 今̊ & 宫竞 & $\dot{\vec{\Xi}}$ & $\stackrel{\dot{\theta 0}}{\vec{z}}$ & & & \\
\hline
\end{tabular}


BY HERBERT R. MORGAN.

\begin{tabular}{|c|c|c|c|c|c|c|c|}
\hline \multirow{7}{*}{$\begin{array}{l}\dot{\overrightarrow{1}} \\
\dot{\overrightarrow{1}} \\
\dot{0}\end{array}$} & B & 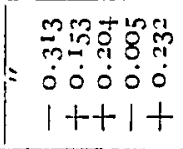 & $\begin{array}{l}80 \% 95 \\
000 \% \\
1+1+1\end{array}$ & 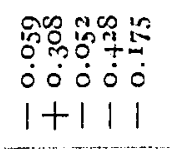 & 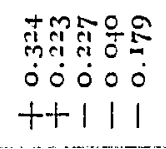 & 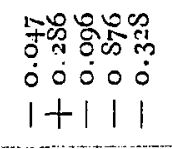 & $\begin{array}{l}50 \\
000 \\
000 \\
000 \\
+11 \\
\end{array}$ \\
\hline & 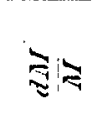 & 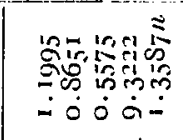 & 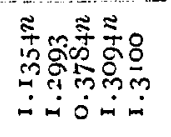 & 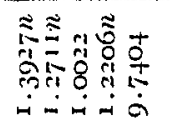 & 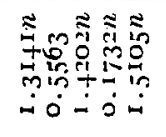 & 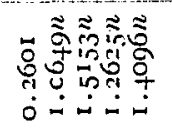 & 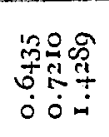 \\
\hline & $\begin{array}{l}D \\
8 \\
8 \\
i\end{array}$ & 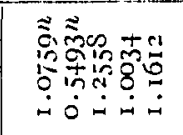 & 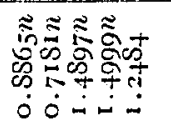 & 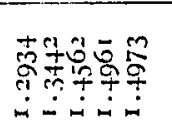 & 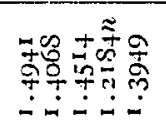 & 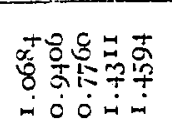 & 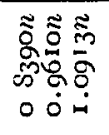 \\
\hline & $\begin{array}{l}D \\
\frac{5}{5} \\
i\end{array}$ & 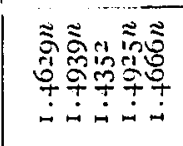 & 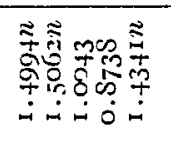 & 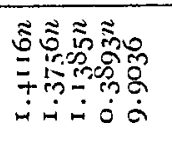 & 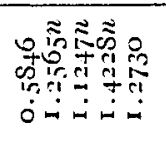 & 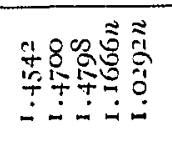 & 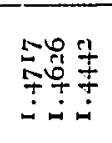 \\
\hline & $\begin{array}{l}1: \\
0 \\
8 \\
0\end{array}$ & 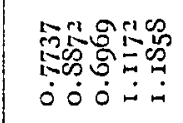 & 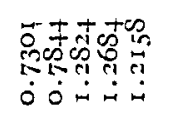 & 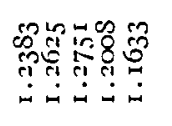 & 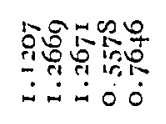 & 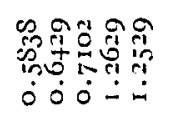 & 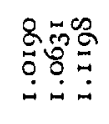 \\
\hline & $\underset{0}{1:}$ & 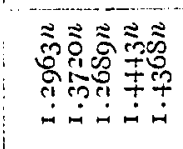 & 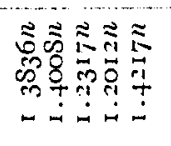 & 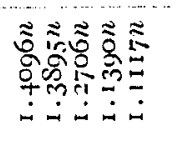 & 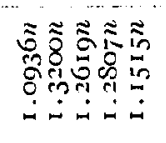 & 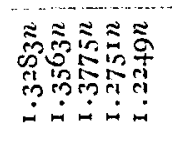 & 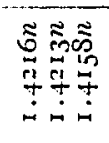 \\
\hline & $\stackrel{\mathbb{N}}{*}$ & 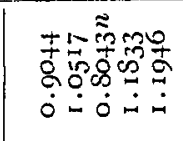 & 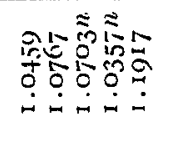 & 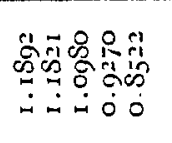 & 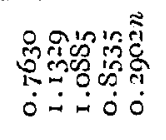 & 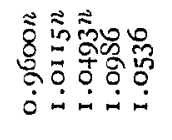 & 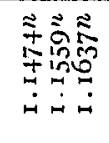 \\
\hline \multirow{6}{*}{ 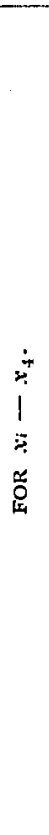 } & is & 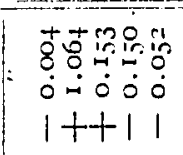 & 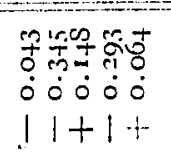 & 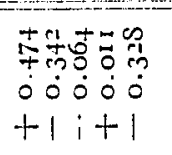 & 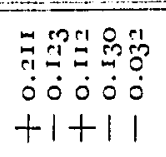 & 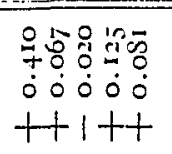 & 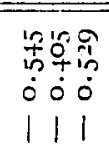 \\
\hline & $\underset{\mathrm{s}}{-}$ & 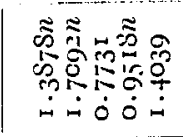 & 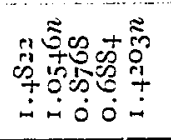 & 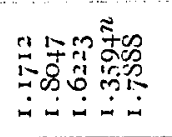 & 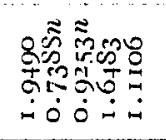 & 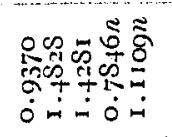 & 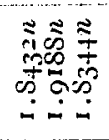 \\
\hline & $\begin{array}{l}1: \\
w \\
0 \\
0 \\
0\end{array}$ & 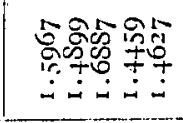 & 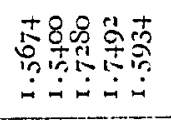 & 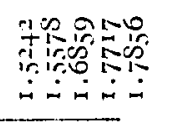 & 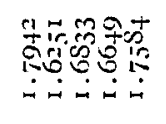 & 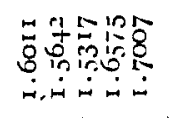 & 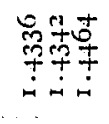 \\
\hline & $\begin{array}{l}1: \\
. \\
0 \\
0\end{array}$ & 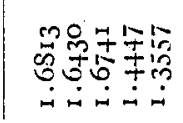 & 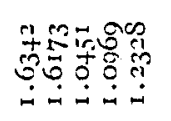 & 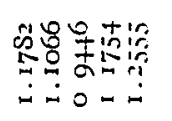 & 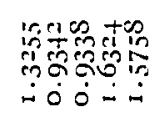 & 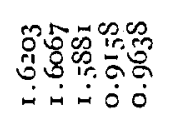 & 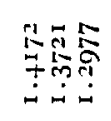 \\
\hline & $\stackrel{1}{=}$ & 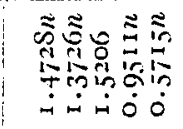 & 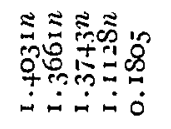 & 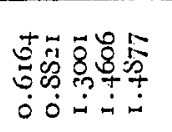 & 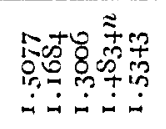 & & \\
\hline & ळ્ & 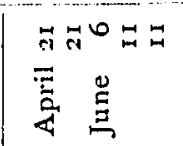 & $\begin{array}{c}m_{m-1}+ \\
\vec{\Xi}\end{array}$ & 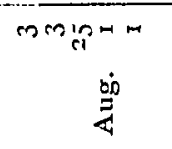 & $\operatorname{tin} \sin \theta \infty$ & & \\
\hline
\end{tabular}



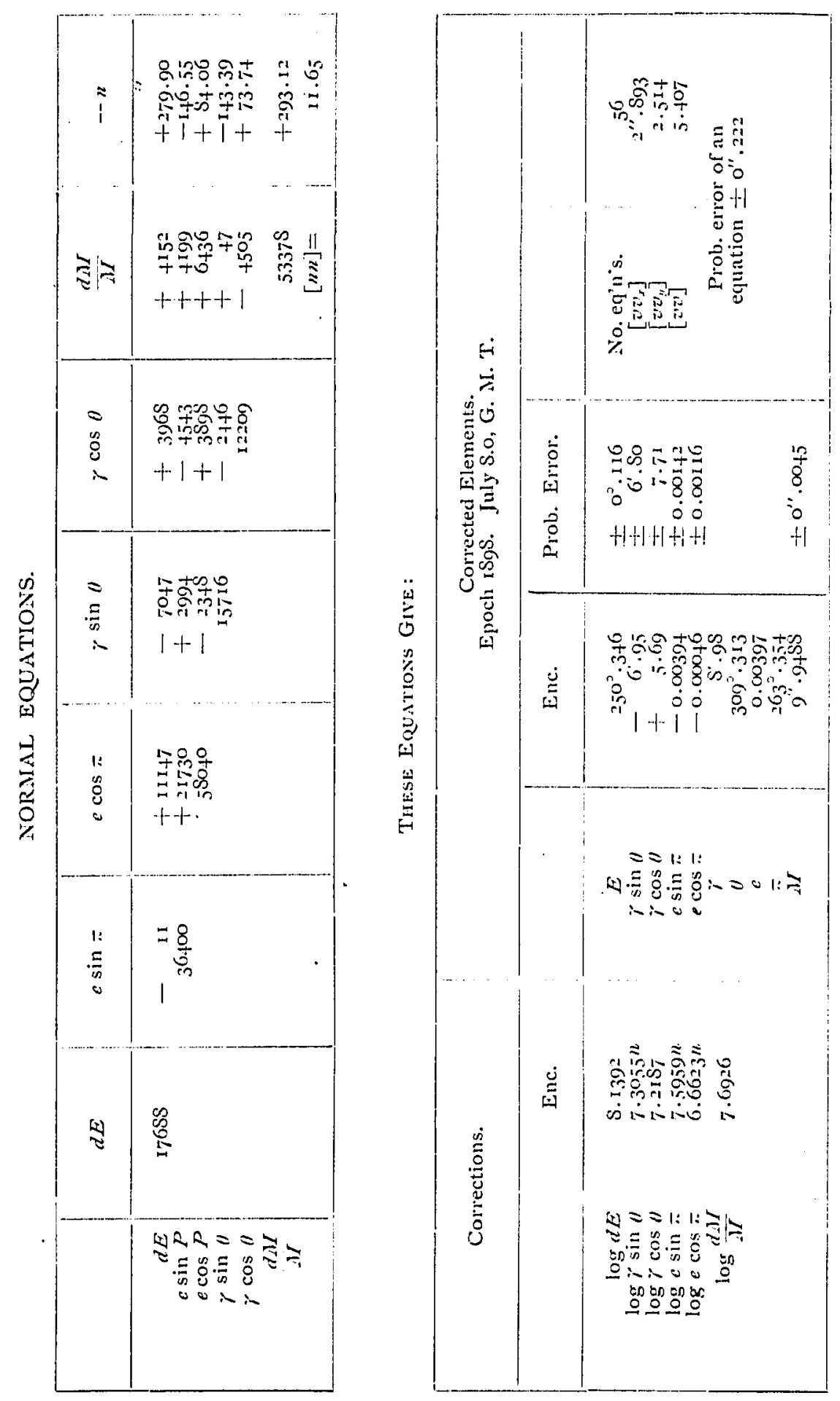
BI HERBERT R. MORGAN.

\begin{tabular}{|c|c|c|c|c|}
\hline $\begin{array}{l}u \\
0\end{array}$ & 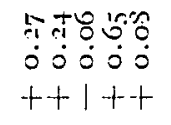 & 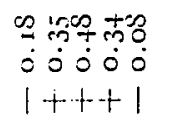 & 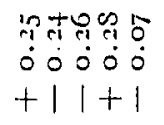 & 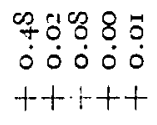 \\
\hline $\overrightarrow{0}$ & 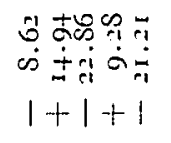 & 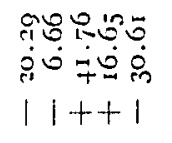 & 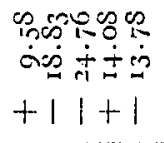 & 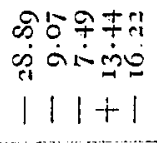 \\
\hline$\dot{\bar{\Xi}}=$ & 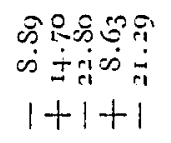 & 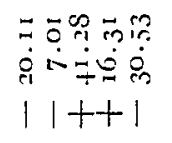 & 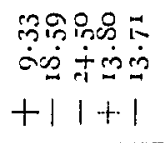 & 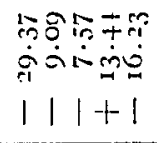 \\
\hline$=$ & 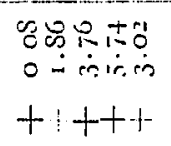 & 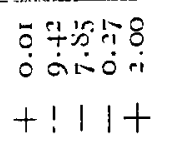 & 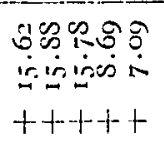 & $\begin{array}{l}\text { qow } 891 \\
\text { inesing } \\
+1111\end{array}$ \\
\hline 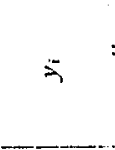 & $\begin{array}{l}50+1 \\
\text { so } 0=0 \\
1+1+1\end{array}$ & 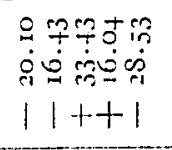 & 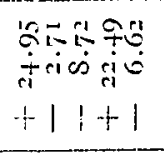 & 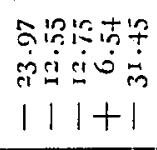 \\
\hline $\begin{array}{l}0 \\
1 \\
0\end{array}$ & $\begin{array}{l}-80 \% \\
100 \\
0000 \\
+111+1\end{array}$ & 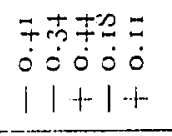 & 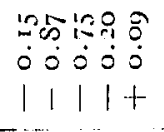 & 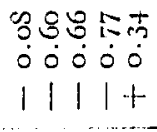 \\
\hline 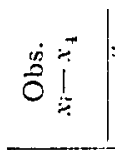 & 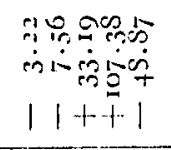 & $\begin{array}{l}0+00 \\
1+1+1 \\
1+000 \\
1+11\end{array}$ & 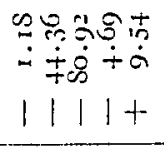 & 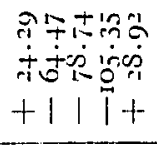 \\
\hline 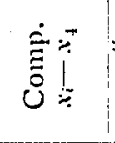 & 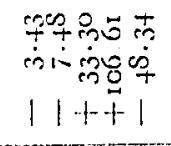 & $\begin{array}{l}10005 \\
30000 \\
1++11\end{array}$ & 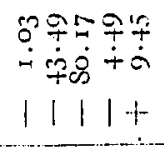 & 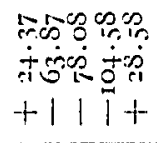 \\
\hline$\because$ & 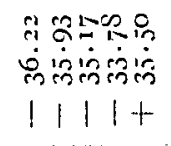 & 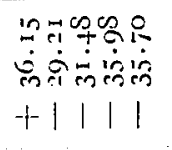 & 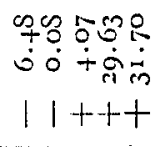 & 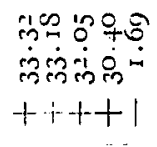 \\
\hline 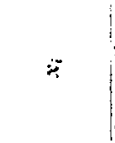 & 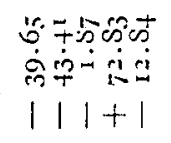 & 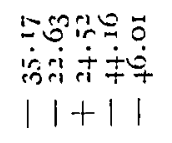 & 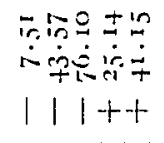 & 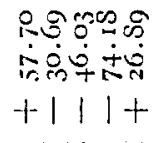 \\
\hline बท!กำ & 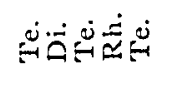 & 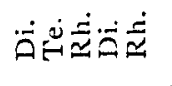 & 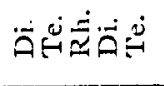 & 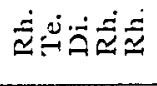 \\
\hline ن. & 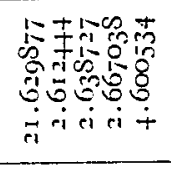 & 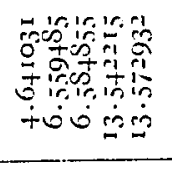 & 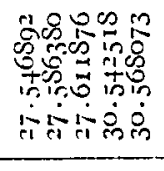 & 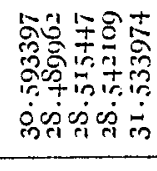 \\
\hline 8 & 异急 & & & $\dot{\dot{g}} \vec{z}$ \\
\hline
\end{tabular}




\begin{tabular}{|c|c|c|c|c|}
\hline & is & 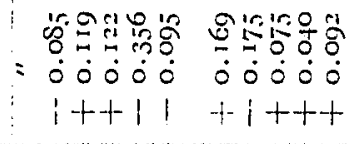 & 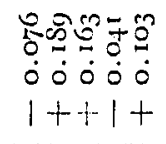 & 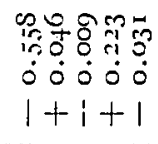 \\
\hline & $\Xi$ & 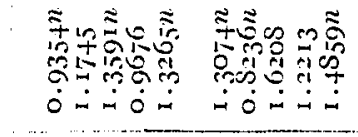 & 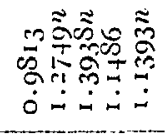 & 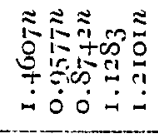 \\
\hline$\rightarrow$ & $\begin{array}{l}= \\
\infty \\
0 \\
0 \\
0\end{array}$ & 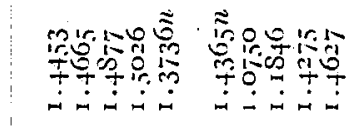 & 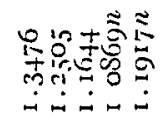 & 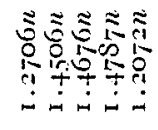 \\
\hline 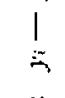 & $\vec{s}$ & 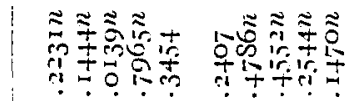 & 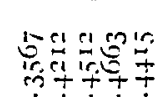 & 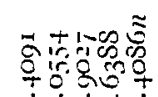 \\
\hline 旁 & 1 & $z-7$ & m & \\
\hline & $\begin{array}{l}1 \\
0 \\
3 \\
0\end{array}$ & 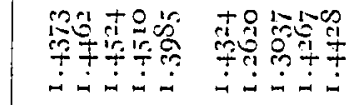 & 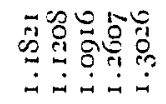 & 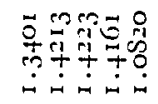 \\
\hline & $\stackrel{:}{:}$ & 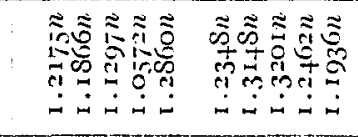 & 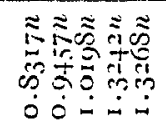 & 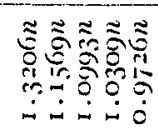 \\
\hline & 斗 & 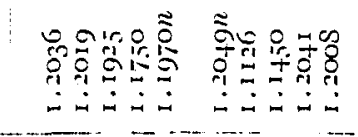 & 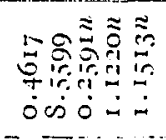 & 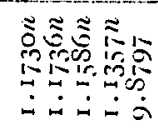 \\
\hline \multirow{6}{*}{$\begin{array}{l}\ddot{\ddot{z}} \\
\approx \\
\ddot{0}\end{array}$} & 2 & 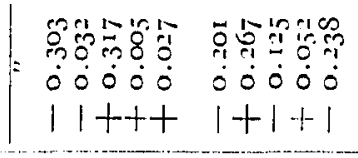 & 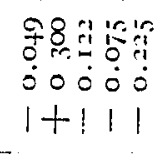 & 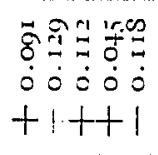 \\
\hline & $\approx$ & 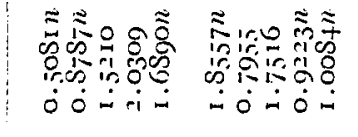 & 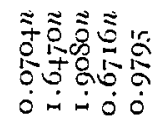 & 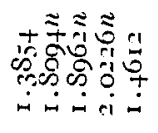 \\
\hline & $\begin{array}{l}1: \\
0 \\
0 \\
0 \\
0\end{array}$ & 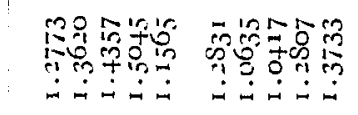 & 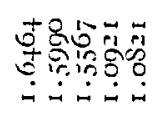 & 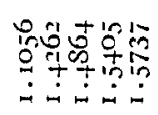 \\
\hline & $\begin{array}{l}1: \\
: \\
0 \\
0\end{array}$ & 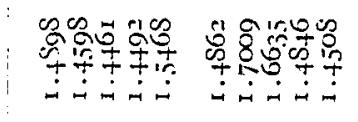 & 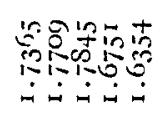 & 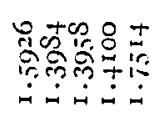 \\
\hline & 约 & 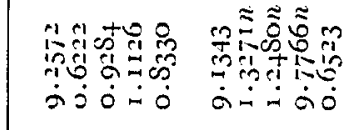 & 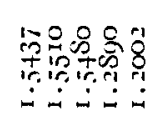 & 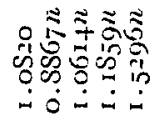 \\
\hline & $\stackrel{8}{\circ}$ & 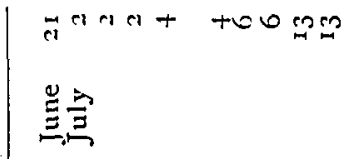 & 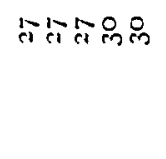 & 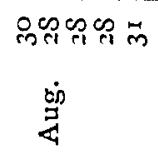 \\
\hline
\end{tabular}


BY HERBERT R. MORGAN.
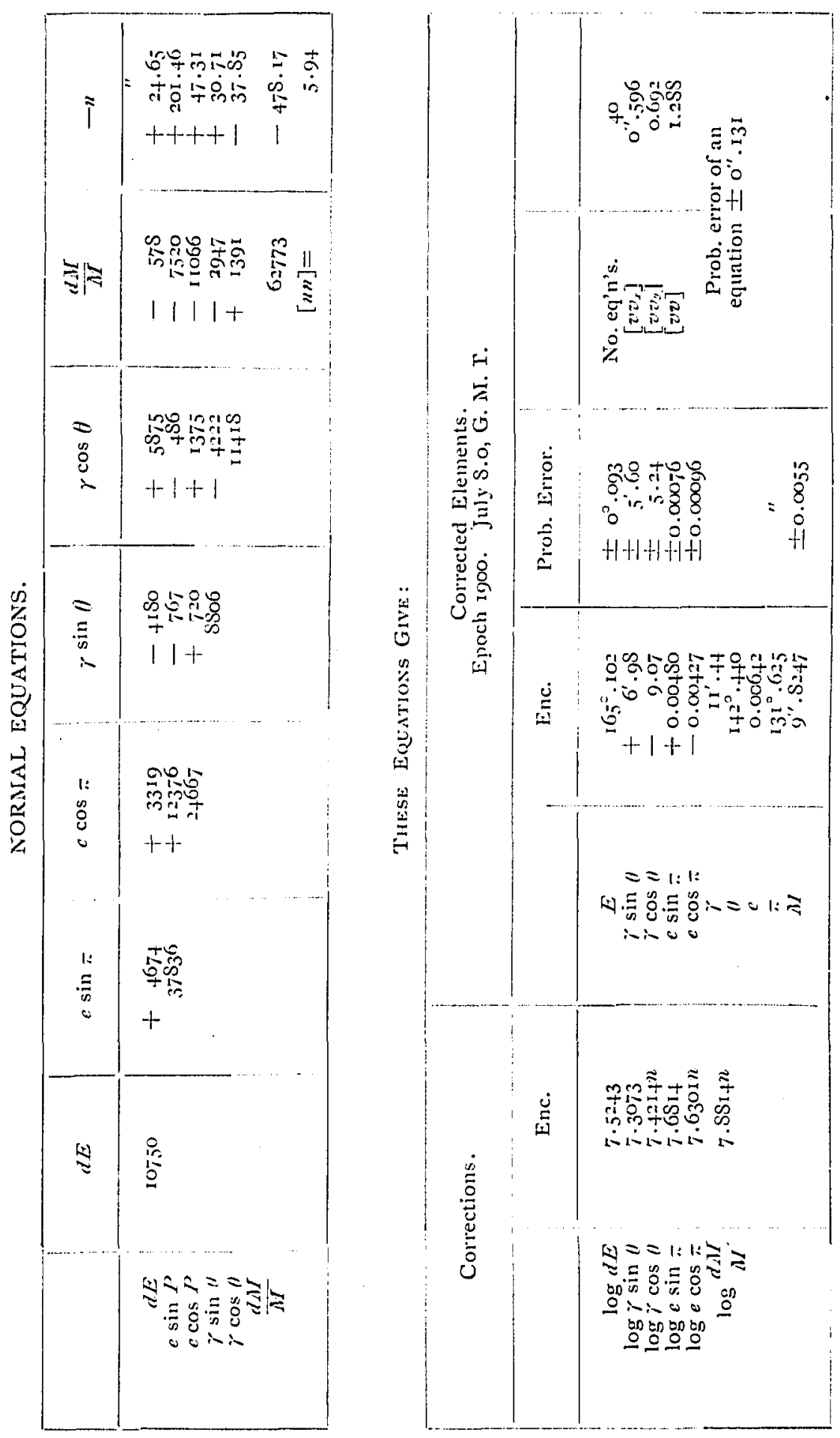
A check solntion was made for 1894 by substitnting, in the normal equations, corrections to the elements of the comparison satellites, and transferring these terms to the ibsolnte terms, and then combining the three sets of normal equations iuto one set, in which the mknown quiutities referred to Enceladus ouly. A solution of this set gave the same as the menn of the three solutions given above. The latter were weighted according to the number of observations. The following are the elements of Enceladus :

$\begin{array}{cccc} & \text { May 8.0, G. M. T. } & \text { July 8.0, G. M. T. } & \text { July } 8.0, \text { G. M. 'T. } \\ & \circ & \circ & \circ \\ E & 331.281 & 250.346 & 165.102 \\ T^{\prime} & 1^{\prime} .5 & 9^{\prime} .0 . & 11^{\prime} .4 \\ \theta & 131^{\circ} .3 & 309^{\circ} .3 & 142^{\circ} .4 \\ e & 0.00581 & 0.00397 & 0.00642 \\ I^{\prime} & 202^{\circ} .1 & 28^{\circ} .9 & 257^{\circ} .2\end{array}$

The menu lougitude for $1889.25+t$, (April 0.0 ), as given by $H$. Struve is

$$
E=199^{\circ} .330+n t+d E . \quad(l=\text { number of days from April } 0.0)
$$

where:

$$
\begin{gathered}
d E=+11^{\prime} .24 \sin \left(143^{\circ}+92^{\circ} .4 t\right)+20^{\prime} .0 \sin \left(75^{\circ}+29^{\circ} .3 t\right) \\
(t=\text { number of years from 1889.25.) }
\end{gathered}
$$

Heace we have, assuming this valne of $d E$, and $u=262^{\circ} .732$ :

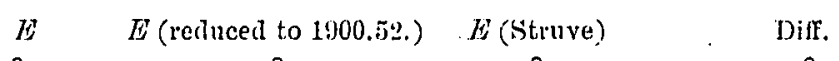

$\begin{array}{ccccr}1894.35 & 331.281 & 164.161 & 164.679 & -0.518 \\ 1898.52 & 250.346 & 164.968 & \text { “ } & +0.289 \\ 1900.52 & 165.102 & 164.682 & \text { “ } & +0.003\end{array}$

The agreement for 1900 is very close, but the difference for 1894 may indicate that there are other terms to be considered in $d . E$.

The eccentricity, $e=0.0058$, is a little larger than that found before.

The longitudes of perisaturnium, 1 , agree with the theoretical motion given by Siruve: $J P=\left(2 n_{1}-n\right)=+123^{\circ} .4$, per year; where $n_{1}$ is the mean motion of Dione.

The inclination, $\not$, being a small quantity, the longitudes of the node, (1, are uncertain, but agree approximately with the theoretical motion, $J(1)=-\beta n=-152^{\circ} .7$, per year; $\beta$ being a function of the constants $e_{i}, n_{i}$, and $J_{i}$, and of the masses, $m_{i}$.

These results, then, are only another proof of the excellence, in theory and observation, of Struve's work.

UNIVERSITY OF VIRGINIA, December 31, 1900. 